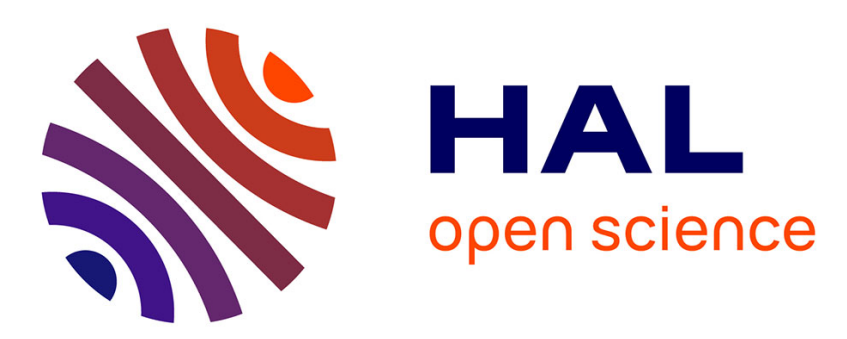

\title{
Identification of mode couplings in nonlinear vibrations of the steelpan
}

Mélodie Monteil, Olivier Thomas, Cyril Touzé

\section{To cite this version:}

Mélodie Monteil, Olivier Thomas, Cyril Touzé. Identification of mode couplings in nonlinear vibrations of the steelpan. Applied Acoustics, 2015, 89, pp.1-15. 10.1016/j.apacoust.2014.08.008 . hal-01084240

\author{
HAL Id: hal-01084240 \\ https://hal.science/hal-01084240
}

Submitted on 18 Nov 2014

HAL is a multi-disciplinary open access archive for the deposit and dissemination of scientific research documents, whether they are published or not. The documents may come from teaching and research institutions in France or abroad, or from public or private research centers.
L'archive ouverte pluridisciplinaire HAL, est destinée au dépôt et à la diffusion de documents scientifiques de niveau recherche, publiés ou non, émanant des établissements d'enseignement et de recherche français ou étrangers, des laboratoires publics ou privés. 


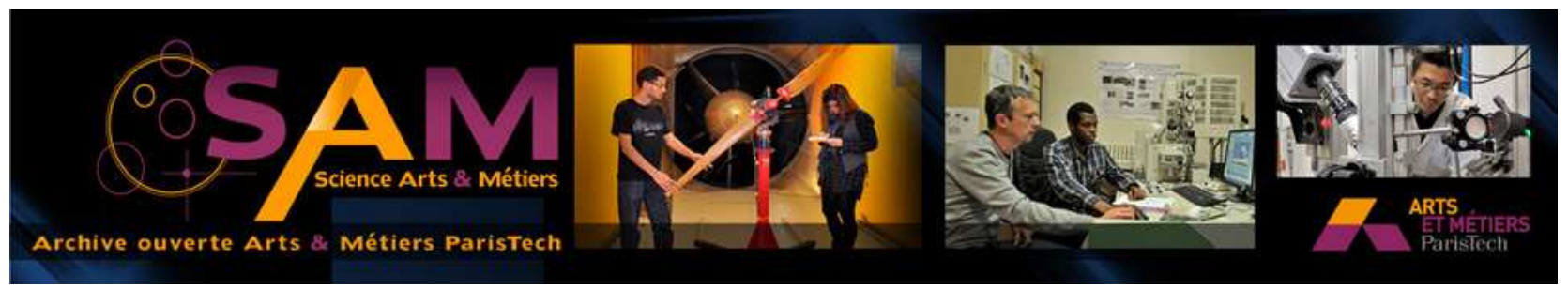

Science Arts \& Métiers (SAM)

is an open access repository that collects the work of Arts et Métiers ParisTech researchers and makes it freely available over the web where possible.

This is an author-deposited version published in: http://sam.ensam.eu

Handle ID: .http://hdl.handle.net/10985/8943

\section{To cite this version :}

Mélodie MONTEIL, Olivier THOMAS, Cyril TOUZÉ - Identification of mode couplings in nonlinear vibrations of the steelpan - Applied Acoustics - Vol. 89, p.1-15 - 2015 


\title{
Identification of mode couplings in nonlinear vibrations of the steelpan
}

\author{
M. Monteil ${ }^{\mathrm{a}, \mathrm{b}, *}$, O. Thomas ${ }^{\mathrm{c}, \mathrm{d}}, \mathrm{C}$. Touzé ${ }^{\mathrm{a}}$ \\ ${ }^{a}$ Unité de Mécanique (UME), ENSTA-ParisTech, 828 Boulevard des Maréchaux, 91762 Palaiseau Cedex, France \\ ${ }^{\mathrm{b}}$ Institut Jean Le Rond d'Alembert, UPMC-Paris VI/CNRS, 4 place Jussieu, 75252 Paris Cedex 05, France \\ ${ }^{c}$ Laboratoire des Sciences de l'Information et des Systèmes, UMR 7296, Arts et Métiers ParisTech, 8 Boulevard Louis XIV, 59000 Lille, France \\ ${ }^{\mathrm{d}}$ Structural Mechanics and Coupled Systems Laboratory, Conservatoire National des Arts et Métiers (CNAM), 2 rue Conté, 75003 Paris, France
}

\section{Keywords:}

Steeldrum

Nonlinear vibration

Internal resonance

Shell

Mode coupling

Energy exchange

\begin{abstract}
A B S T R A C T
The vibrations and sounds produced by two notes of a double second steelpan are investigated, the main objective being to quantify the nonlinear energy exchanges occurring between vibration modes that are responsible of the peculiar sound of the instrument. A modal analysis first reveals the particular tuning of the modes and the systematic occurence of degenerate modes, from the second one, this feature being a consequence of the tuning and the mode localization. Forced vibrations experiments are then performed to follow precisely the energy exchange between harmonics of the vibration and thus quantify properly the mode couplings. In particular, it is found that energy exchanges are numerous, resulting in complicated frequency response curves even for very small levels of vibration amplitude. Simple models displaying 1:2:2 and 1:2:4 internal resonance are then fitted to the measurements, allowing to identify the values of the nonlinear quadratic coupling coefficients resulting from the geometric nonlinearity. The identified 1:2:4 model is finally used to recover the time domain variations of an impacted note in normal playing condition, resulting in an excellent agreement for the temporal behaviour of the first four harmonics.
\end{abstract}

\section{Introduction}

Steelpans are musical percussion instruments coming from the island of Trinidad and Tobago in the Caribbean. They are traditionally played in orchestras called steelbands. These orchestras are composed of several steelpans covering a range of several octaves. Each steelpan is made of an oil barrel that is subjected to several stages of metal forming that stretch and bend the structure. The top of the barrel is pressed, hammered, punched and burnt in order to obtain a sort of main bowl within which convex domes are formed, each one corresponding to a particular musical note (Fig. 1). These notes are associated to vibration modes with strongly localized shapes on the domes, that are finely tuned in harmonic relationships by the tuner. As a consequence, when struck, each dome vibrates with a quasi-harmonic spectrum responsible of a musical note with a clear pitch. In all cases, the second mode is tuned at twice the frequency of the fundamental one, whereas the third one is tuned at three or four times the fundamental, depending on the note and on the tuner. Higher

* Corresponding author at: Unité de Mécanique (UME), ENSTA-ParisTech, 828 Boulevard des Maréchaux, 91762 Palaiseau Cedex, France. Tel.: +33 1693197 34; fax: +33169319997.

E-mail address: melodie.monteil@gmail.com (M. Monteil). frequency modes are also present, but no general rule exists for their tuning [1].

The vibrational behaviour of the steelpan has been studied for some times and numerous results are already available in literature. The seminal investigations are due to Achong who produced a series of papers in the 1990's that describe the most important physical phenomena: mode localization, nonlinear vibrations, mode couplings and energy exchanges [2-5]. All these features are now widely recognized as being the key to properly understand the vibrational behaviour of steelpans and their peculiar sound. Mode localization allows creating different notes relying on the same structure, while nonlinear geometric nonlinearity is responsible of energy exchanges and thus on the peculiar tone of the pan. Modal analyses have been performed by Rossing et al. in different papers [6-8], underlining that modes are perfectly tuned during the manufacturing process. In particular, the tuner takes care of adjusting the first modes of each note so that one mode is an octave above the fundamental, while a third one is tuned either a twelfth or two octaves above the fundamental $[1,7]$. These findings have been reinforced by other measurements showing either the sound radiation $[9,10]$, the response to impulsive excitation [11] and the identification of sound sources by time-reversal imaging [12]. On another point of view, detailed metallurgical studies have been realized by Murr, Ferreyra et al. [13-16]. 
(a)

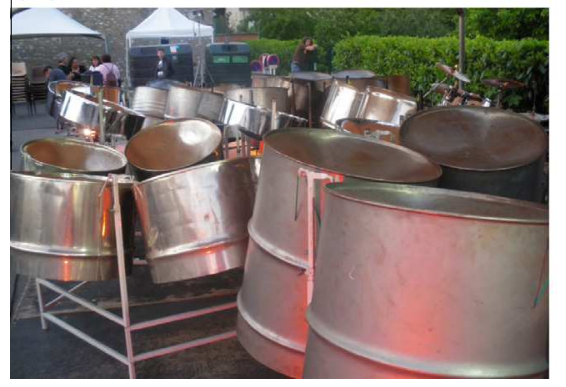

(b)

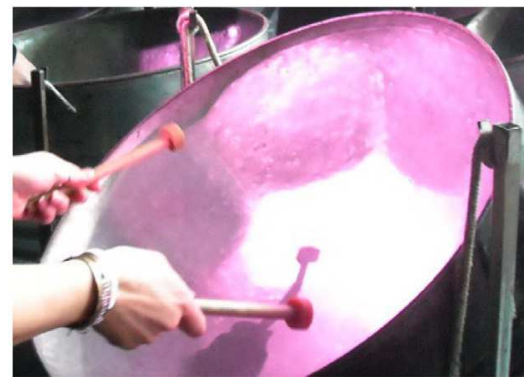

Fig. 1. (a) Photograph of a steel band. (b) Photograph of a particular steelpan in normal playing conditions.

The effects of thickness reduction, deformation, hardness location and heat treatments have been precisely measured and some innovations in steelpan materials have been proposed [17-19].

The aim of the present paper is to provide new results and insight into the linear and nonlinear vibrations of the steelpan. Two notes of a double second are specifically studied. The modal analysis reveals the systematic occurence of degenerate modes from the second one, a feature that has not been particularly commented to our knowledge. For the nonlinear vibration, a particular emphasis is put on the identification of mode couplings in order to precisely understand the energy exchanges occurring during the vibration. Measurements in forced vibrations are used in order to have a finely tuned and perfectly reproducible experiment for which all input parameters are tightly controlled. Thanks to lockin amplifier, harmonics 1-8 of the vibration signals are separated and followed separately. The measurements obviously reveal the complexity of the vibrational patterns and the number of excited modes, even for very small levels of vibration amplitudes. Simple models displaying 1:2:2 and 1:2:4 internal resonance, available in [20], are then used in order to fit some experimental frequency response curves. Finally, time-domain simulations of the models identified from forced vibrations, are used to compare the time response of the first four harmonics of the signal of an impacted note, showing a perfect agreement.

\section{Modal analysis of the steelpan}

In this section, an experimental modal analysis of the steelpan is presented. The steelpan under study, shown in Fig. 2, is a right barrel of a double second (middle-high frequency steelpan). It is composed of 19 precisely tuned notes, distributed on three concentric circles, the lower notes being on the outer circle. As we focus on the nonlinear response of two different notes, namely G3 and F3, two different modal analysis have been performed, by successively exciting directly the studied note, and by measuring with a scanning laser vibrometer the notes along with its two harmonically tuned neighbours (G4 and G5 for the G3 note, and F4, F5 for the F3 note). As already known from available studies [2,3,7-9], and confirmed by a complete modal analysis performed on the same instrument in our laboratory [21], the vibrations are localized so that scanning the wole surface is not needed (see Fig. 3(b)).

In the experiments, a pointwise excitation is ensured by a coil/ magnet exciter. This home-made device has already been used in numerous experiments on nonlinear vibrations of plates and shells $[22,23]$. In particular, the interested reader can find in [22] a complete study on the calibration and the performance of the device, showing in particular that it allows for a clean harmonic excitation with a very low harmonic distortion due to the nonlinearities of the system. In particular it behaves far better than a classical

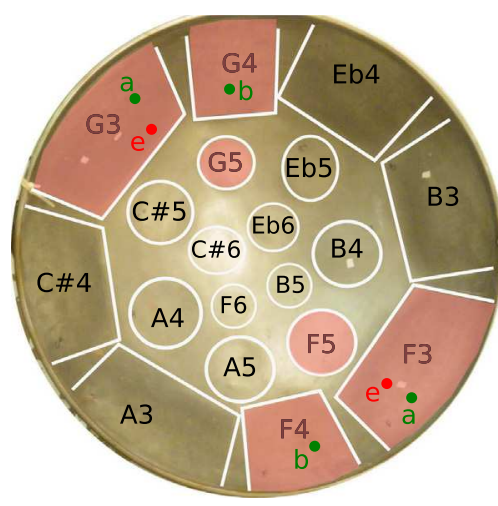

Fig. 2. Pattern of the steelpan under study, and selected notes scanned by the vibrometer during the modal analysis. Excitation points on G3 and F3 are denoted e, while $a$ and $b$ refers to the selected points for measuring the transverse displacement. The scanned regions for the mode shapes identifications are pinkshaded. (For interpretation of the references to color in this figure legend, the reader is referred to the web version of this article.)

shaker by ensuring a total harmonic distortion less than $2 \%$ [22]. In the experimental set-up, no force transducer is used: the current sent to the coil is measured and a calibration procedure allows us to measure the proportionality coefficient $K$ between the current (in A) and the force (in N). For our set of experiments, the measurement gives $K=0.1 \mathrm{~N} / \mathrm{A}$. Fig. 3(a) shows how the device is placed below the steelpan.

For the modal analysis, the excitation device is fed with a swept sine in the frequency band $[0,1700] \mathrm{Hz}$. A Polytec PSV-300 scanning laser vibrometer is used for measuring the velocity at a given grid point. Fig. 3(b) shows the operational deflection shape when the G3 note is excited at $197.5 \mathrm{~Hz}$, underlining the fact that the vibration is, for this frequency, strongly localized to the G3 dome only.

Fig. 4 shows the frequency response function (FRF) in velocity with respect to the prescribed force at the excitation point, when the steelpan is excited on the note G3. The first mode is identified at $197.5 \mathrm{~Hz}$. Harmonic relationships are exhibited by dashed lines. It can be observed that around $2 f_{1}$, the frequency spectrum presents a double peak indicating that the mode is degenerate, with two mode shapes having nearly equal frequencies. The next mode is located in the vicinity of the harmonic relationship $4 f_{1}$, then the higher eigenfrequencies are significantly shifted as compared to a perfect tuning $\left(\simeq 6 f_{1}, \simeq 8 f_{1}\right)$.

Two local views around $2 f_{1}$ and $4 f_{1}$ are presented Fig. 4(a) and (b) respectively. The FRF of the excited point e (black line) and of point $b$, located in the vicinity of the center of the G4 note (red dashed line), are superimposed (see Fig. 2 for the points locations). By changing the measurement point, one clearly reveals that degenerate modes are present both around $2 f_{1}$ and $4 f_{1}$. 

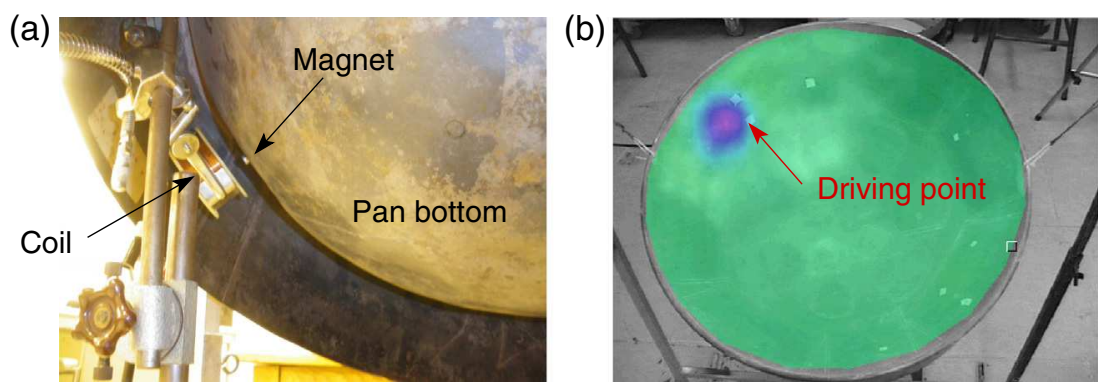

Fig. 3. Modal analysis of the double second. (a) Electromagnetic exciter. (b) Mode shape with energy localized on the excited note of frequency 197.5 Hz.
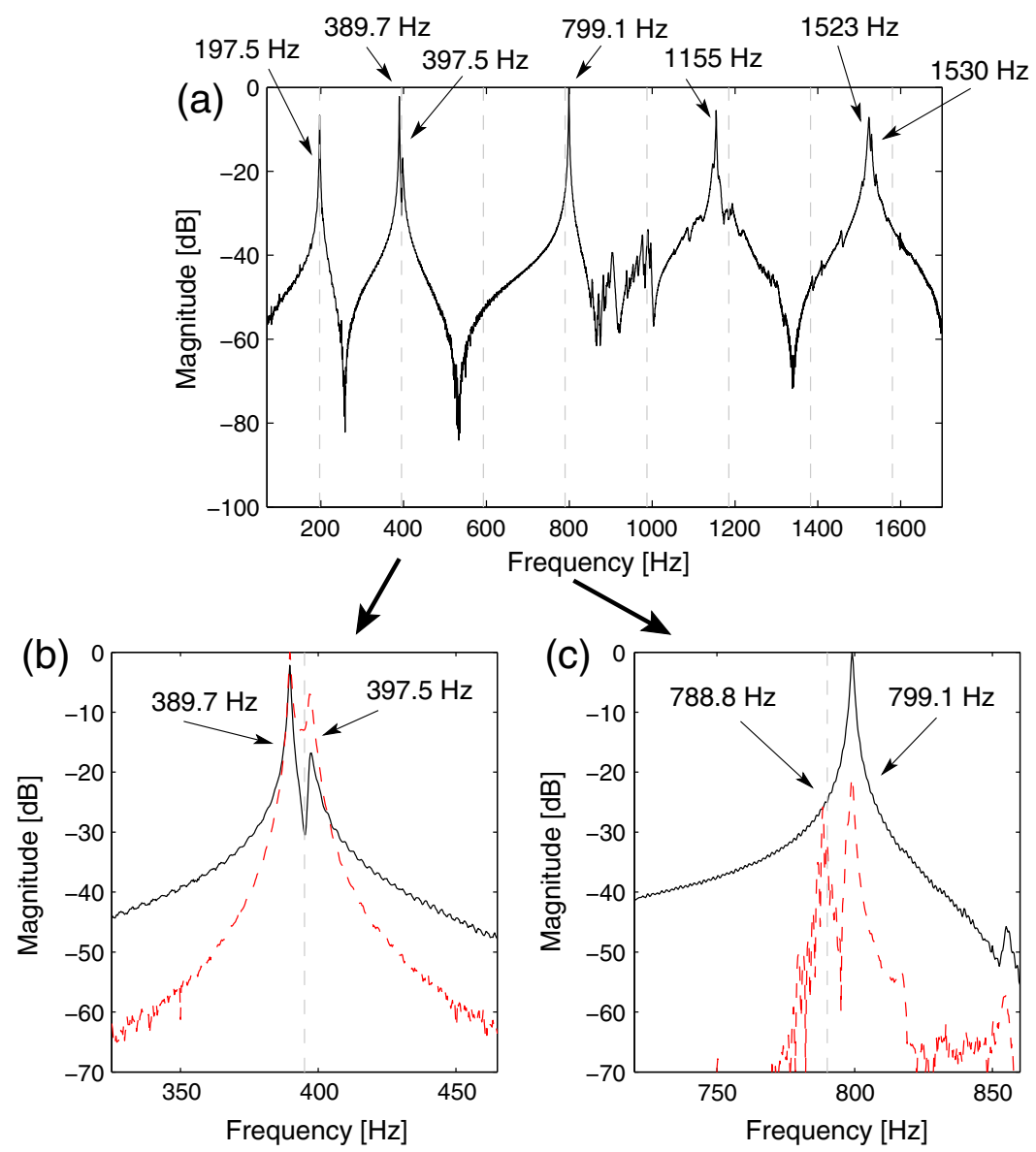

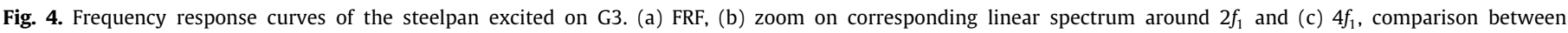

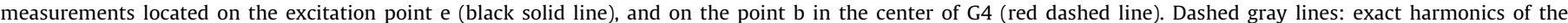

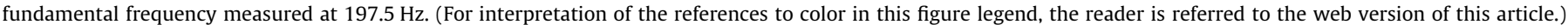

The results for the second experiment, for which the F3 note is excited, are reported on Fig. 5. Fig. 5(a) shows that the first three eigenmodes are also tuned at $f_{1}, 2 f_{1}$ and $4 f_{1}$, where $f_{1}$ is identified at $172.2 \mathrm{~Hz}$. Fig. 5(b) and (c) precise the presence of double peaks, revealed when the FRF is measured on the note $\mathrm{F} 4$ around $2 f_{1}$ and $4 f_{1}$. In this case, the higher-frequency eigenmodes are also shifted as compared to the perfect tuning. The spectrum repartition is around $\simeq 5 f_{1}, \simeq 7 f_{1}$ and $\simeq 9 f_{1}$.

Figs. 6 and 7 summarize the linear characteristics obtained for the two notes from the modal analysis: eigenfrequencies, modal damping factors and associated operational deflection shapes at resonances are given. In both cases, around $f_{1}$, the vibrational pattern is localized on the center of the note area. This mode, having no nodal diameters, is commonly denoted $(0,0)$. The degenerate modes around $2 f_{1}$ are then shown. Their vibrational pattern is composed of the shape $(0,1)$ with a vertical nodal line on the principal note, accompanied with a shape $(0,0)$ on the harmonically tuned note (G4 and F4). The shape on this auxiliary note is either in-phase, or out-of-phase, and a small detuning between the two frequencies is observed. This degeneracy of modes is a common feature in systems presenting localized vibrations. In particular, one can find in [24] how this degeneracy appears for a one-dimensional beam resting on an elastic foundation with varying stiffness along the position with a symmetric profile. In the present case of the steelpan, this degeneracy is a consequence of both the particular harmonic tuning of contiguous notes G3-G4 (or F3-F4) and the mode shape localization.

The phenomenon repeats for the two modes appearing around $4 f_{1}$, where the shape on the principal note is $(1,0),(0,1)$ on the harmonic G4 (or F4) note, and $(0,0)$ on the second harmonically tuned 

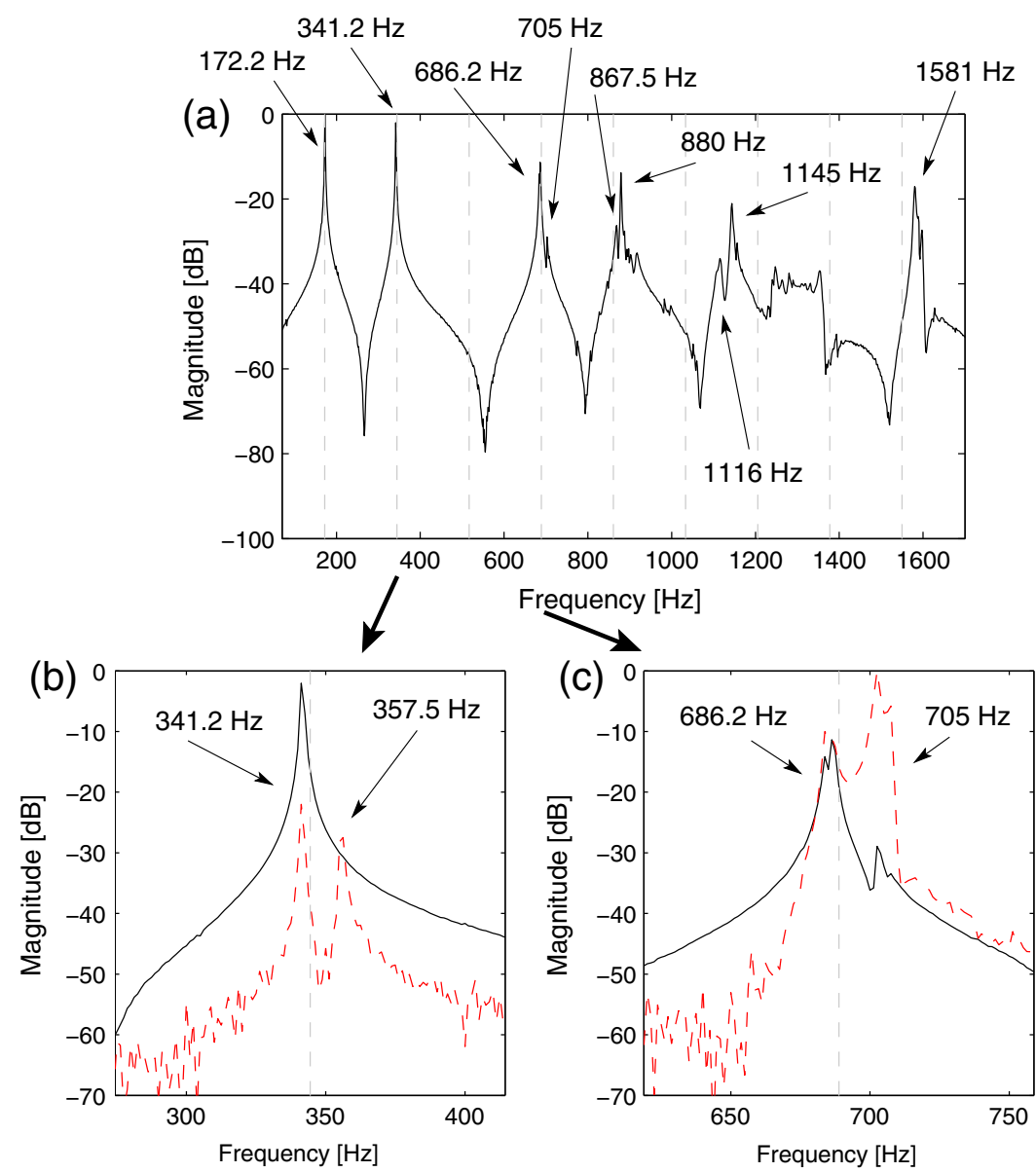

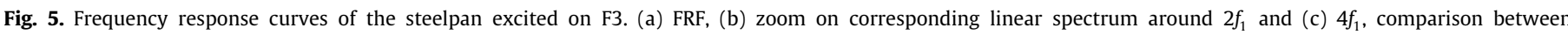

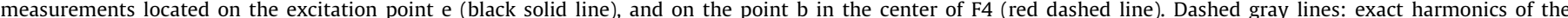

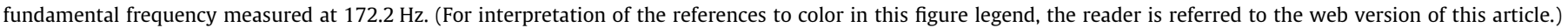

note G5 (or F5). Theoretically, as now the mode expands on three contiguous domes, one would expect to obtain a double degeneracy for the modes around $4 f_{1}$, as the similar process observed for the note at $2 f_{1}$ can now produce four different patterns. However, the quadruplets may be difficult to measure for some physical reasons (difficulty to excite some of the modes, frequency relationship not perfectly fitted, etc.).

For the note G3, the measurement at point b shown in Fig. 4(c) shows that secondary peaks are present around $788.8 \mathrm{~Hz}$. This could indicates the presence of a degeneracy with eigenfrequencies very close to the mode identified at $788.8 \mathrm{~Hz}$ and shown in Fig. 6. One can note however that the secondary peaks appear to be very small in amplitude, which means that these two configurations are difficult to measure and to excite in our experimental set-up, for physical reasons due to imperfections of the shell. Moreover, the operational deflection shapes in Fig. 6 show that the pattern is not so perfectly reproducible as for the degenerate modes at $2 f_{1}$, rendering the whole identification of the possible quadruplets very difficult. Hence, in the remainder of the paper, we have decided to extract and identify only the main peaks where most of the vibrational energy is contained, so that only two modes are shown in Fig. 6 at $4 f_{1}$.

For the note F3, the measurements in Fig. 5(c) at points a and b show respectively the presence of a double peak around $686.2 \mathrm{~Hz}$, as well as a double peak around $705 \mathrm{~Hz}$, thus indicating that the double degeneracy at $4 f_{1}$ is also observable. For the same reasons as for the note $\mathrm{G} 3$, we have however decided to identify and extract only the main vibratory components, so that only two modes around $4 f_{1}$ are presented in Fig. 6. These choice have also been guided by the fact that in forced vibration experiments shown in the next section, these modes have not been observed.

For the higher frequency modes, the vibrational patterns complexifies and the order of appearance of mode shapes differs for the two studied notes. For the note G3, a bunch of frequency peaks is observed around $5 f_{1}$, whose modes are difficult to separate. The shape of one of them, shown on Fig. 7, shows a $(0,2)$ pattern on the principal note and a $(1,0)$ pattern on the harmonic G4. For the same note, a $(1,1)$ shape is observed around $6 f_{1}$ and two degenerate $(2,1)$ are measured around $8 f_{1}$. For the note $\mathrm{F} 3$, two degenerate modes are present around $5 f_{1}$ with a $(1,1)$ shape, and around $6 f_{1}$ with a $(0,3)$ modal shape. Finally a $(0,4)$ is also observed around $8 f_{1}$.

This modal analysis reveals the strong localization phenomenon present in steelpan vibrations, and already underlined in previous investigations. A consequence of this localization is the presence of degenerate modes in the case of harmonically tuned contiguous notes, from the second frequency, where in-phase and out-of-phase components can be present in the auxiliary pattern. Consequently, from the second eigenfrequency, eigenmodes are most likely to appear at least by pair. For the two studied notes, the first eigenfrequencies appear in a ratio $f_{1}, 2 f_{1}, 4 f_{1}$, denoted in the remainder of the paper 1:2:4 relationship. As the steelpan's shell is particularly thin (of the order of $0.5 \mathrm{~mm}$ in the center of the notes), geometrically nonlinear vibrations are excited in normal conditions of playing. The fact that those $1: 2: 4$ relationships are present implies that internal resonance conditions will be 


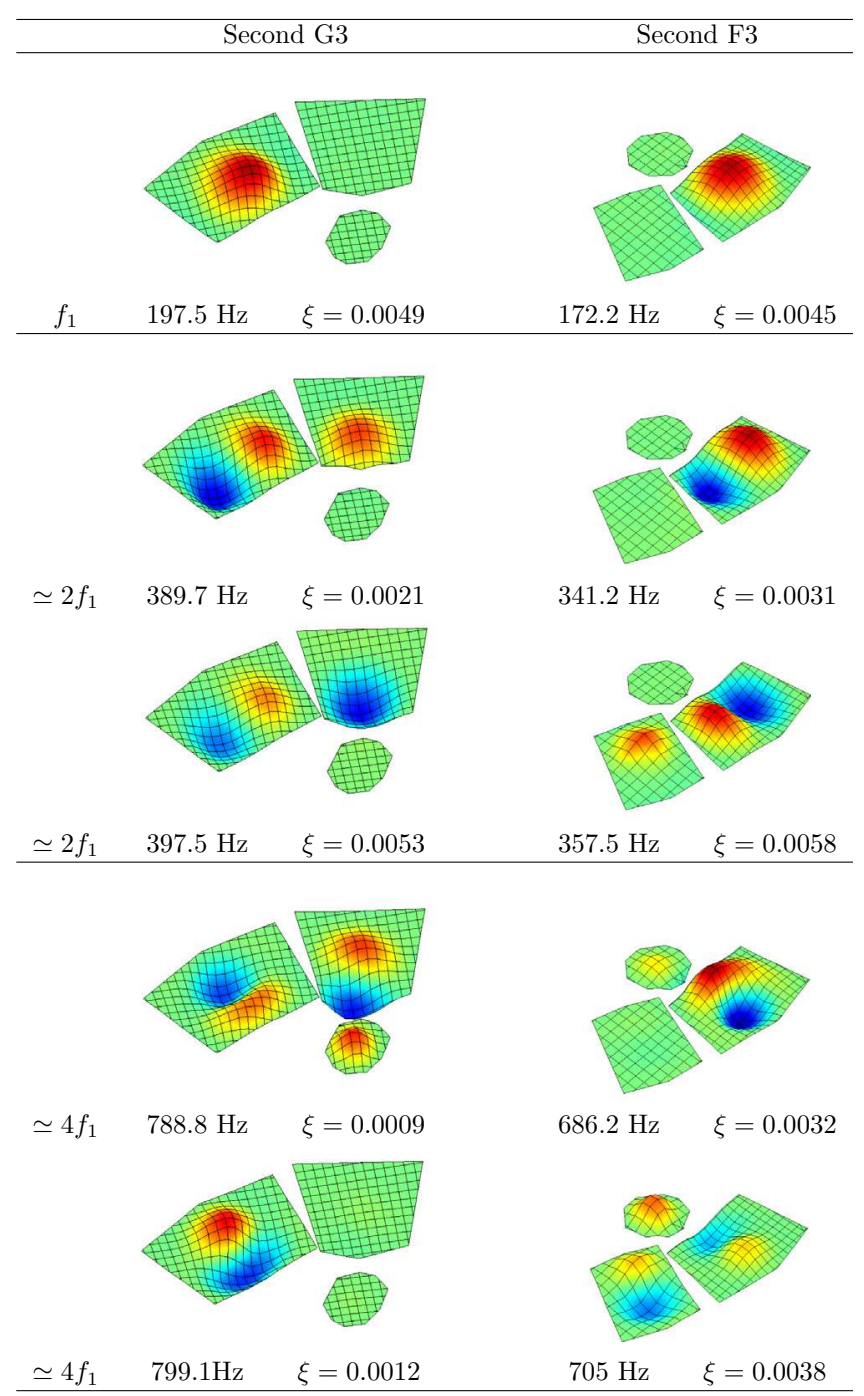

Fig. 6. Operational deflection shapes, eigenfrequencies and modal damping ratio, for the notes G3 and F3. Fundamental mode $\left(f_{1}\right)$; modes around $2 f_{1}$ and $4 f_{1}$. The scanned parts of the upper surface of the steel pan are shown by on Fig. 2 .

easily fulfilled via quadratic nonlinear terms of the model [20,25-27]. Hence energy exchanges between modes will be more easily activated, favoring the transfer of energy to higher frequencies. The remainder of the paper is concerned by an experimental identification of these mode couplings.

\section{Nonlinear interactions in the steelpan vibrations}

In usual playing, the caribbean steelpan is stroke by percussive mallets called sticks, which create free vibrations. In order to properly reveal the nonlinear interactions in the nonlinear dynamics of the steelpan, the instrument will be harmonically excited by a pointwise external force imposed by the coil/magnet system. By increasing gradually the amplitude of the external force and by sweeping the excitation frequency, linear and nonlinear responses will be observed. This reproducible and controlled experimental set-up allows for a fine identification of energy transfers and nonlinear interactions, in the vibration amplitude range of the usual playing. We begin by describing the experimental set-up, then the measurements for the two studied notes, G3 and F3, are reported and commented. In particular, the measurements reveal that for vibration amplitudes that are below the amplitudes

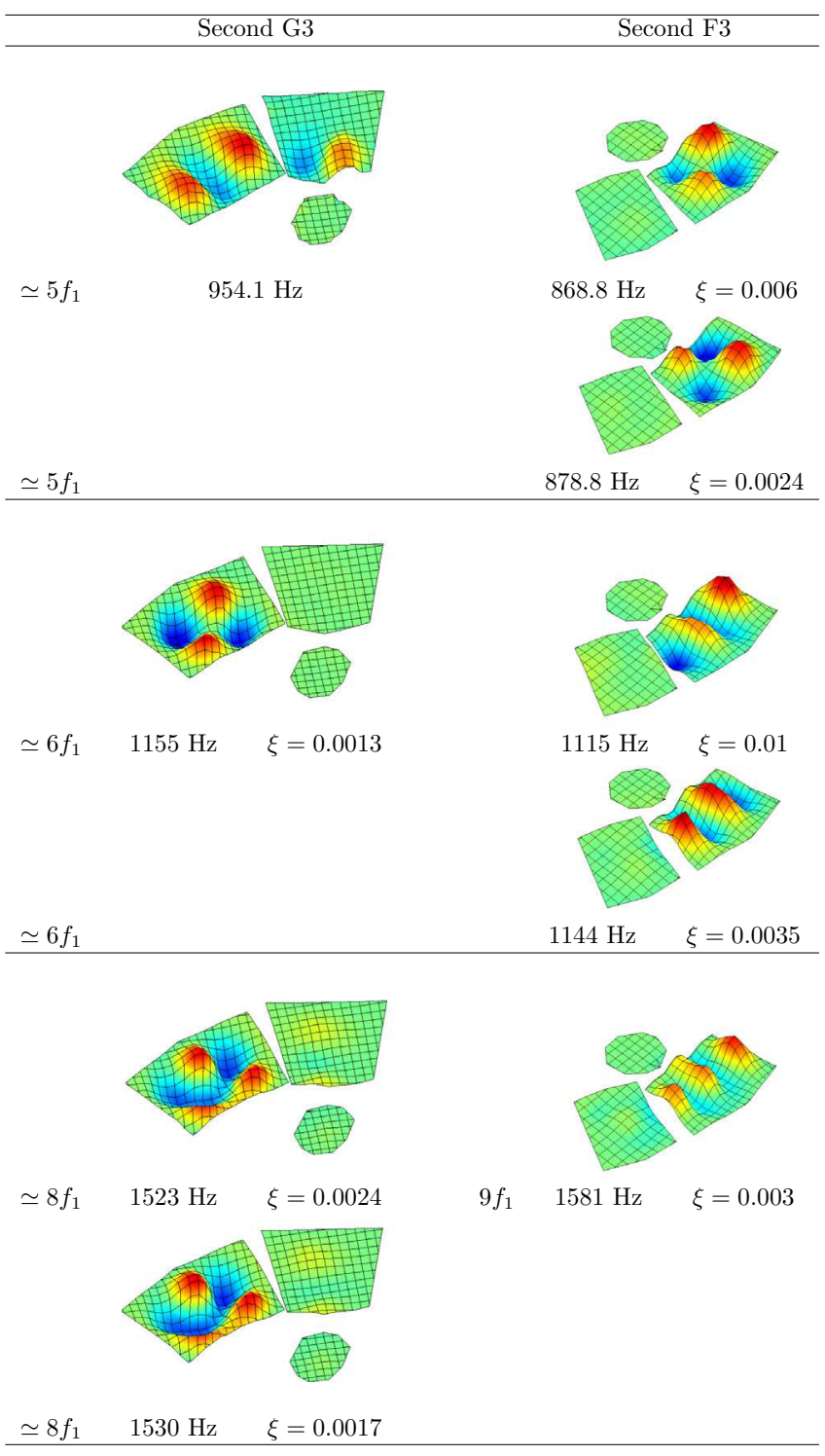

Fig. 7. Operational deflection shapes, eigenfrequencies and modal damping ratio, for the notes G3 and F3 (following Fig. 6). Modes around $5 f_{1}, 6 f_{1}$ and $8 f_{1}$.

observed in usual playing, strong modal interactions and energy exchange already occur.

\subsection{Experimental setup}

The experimental setup is sketched in Fig. 8. The coil/magnet device, already used for the modal analysis, allows injection of the external force, at a given, selected point of the structure. The coil is fed by a sine signal of frequency $f_{d r}=\Omega / 2 \pi$, generated by a low distortion signal synthesizer (a Stanford Research System DS-360). The vibration velocity is measured at a given point by the laser vibrometer, and the harmonics amplitudes are estimated by a lock-in amplifier (a Stanford Research System SR-830). To obtain the displacement of each harmonics, the measured velocity is divided by the angular frequency of the harmonics. The reference signal for the lock-in amplifier is the driving sine signal of frequency $f_{d r}$, or another signal of frequencies $f_{d r} / 2$ or $f_{d r} / 4$, generated by the built-in generator of the device, in the case in which subharmonic of the driving signal have to be measured. For each measurement reported in the paper, the system is excited in a local 


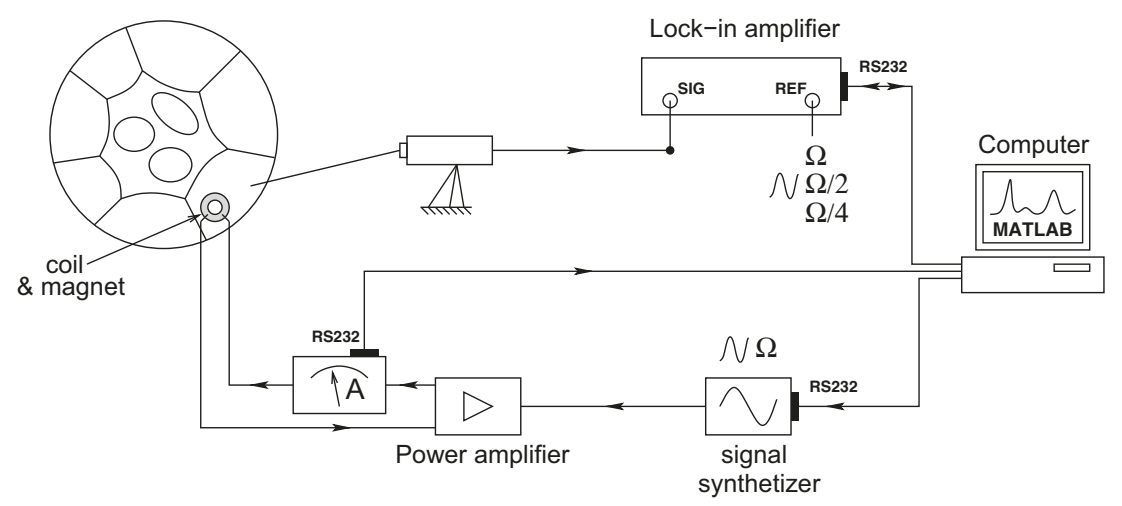

Fig. 8. Experimental setup for measuring the harmonics of the nonlinear frequency response curves.

frequency band around a particular resonance, at a discrete set of driving frequencies that are followed increasingly (forward "sweep") or decreasingly (backward "sweep"). For each point, the driving signal is kept at a constant frequency and a time delay of $4 \mathrm{~s}$ is awaited so as the transient to die out. Then, the amplitudes of each signal harmonics in the steady state are measured during $10 \mathrm{~s}$, thanks to the lock-in amplifier. In those ten seconds, about twenty values for the amplitudes are recorder in order to identify potential quasi-periodic oscillations. After, the frequency is changed and the following point is recorded. A forward as well as a backward sweep are realized for each measurement, to identify jump phenomena, so that the total time of measurement for producing a frequency response curve is approximately three hours. All the measurements and the command of the several devices are automated thanks to RS232 protocols and a Matlab computer program.

Analogous measurements have been performed to identify internal resonances in circular plates [22] and spherical shells [23] previously. The main improvement brought in the present work is the use of a lock-in amplifier and the full automation of the experiments, which allows very precise measurements, in term of frequency resolution, identification of quasi-periodic oscillations and number of measured harmonics (up to four distinct harmonics are measured in a given experiment).

\subsection{Nonlinear response of the note G3}

The first experiment consists in exciting the note $\mathrm{G} 3$ with a driven frequency $f_{d r}$ close to the first three resonances $\left(f_{1}, 2 f_{1}\right.$ and $\left.4 f_{1}\right)$ with a forward and a backward sweep. The amplitudes of several harmonics of the response signal are measured. We begin with the case for which the external frequency is in the vicinity of the first eigenfrequency: $f_{d r} \simeq f_{1}$. For all the figures of this section, readability is helped by inserting vertical markers so as to precisely locate the different eigenfrequencies of the system, as well as their harmonics. The marker symbols for each harmonics are defined in Table 1.

Table 1

Values of the eigenfrequencies identified for note $\mathrm{G} 3$, and marker symbols used in all related figures (Figs. 9-12).

\begin{tabular}{lll}
\hline Frequency relationship & Value $(\mathrm{Hz})$ & Marker symbol \\
\hline$f_{1}$ & 197.5 & $(--)$ \\
$\simeq 2 f_{1}$ & 389.7 & $(-\cdot-)$ \\
$\simeq 4 f_{1}$ & 397.5 & \\
& 788.8 & $(\cdots)$ \\
$\simeq 6 f_{1}$ & 799.1 & $(\cdots)$ \\
\hline
\end{tabular}

\subsubsection{Low frequency excitation: $f_{d r} \simeq f_{1}$}

The amplitude response of the displacement, decomposed on three harmonics oscillating around $f_{1}, 2 f_{1}$ and $4 f_{1}$ and denoted $H_{1}, H_{2}$ and $H_{4}$ respectively, is shown in Fig. 9. The amplitude of the external forcing is measured as the current in the coil $I=2.01 \mathrm{~A}$, which thus corresponds to an amplitude of $0.2 \mathrm{~N}$. For that level of excitation, the amplitude of the first harmonic is small, with a maximum value at $0.04 \mathrm{~mm}$, less than one tenth the thickness. However, the response is already nonlinear as shown by the behaviour of the three measured harmonics. $H_{1}$ presents a maximum at the linear frequency $197.5 \mathrm{~Hz}$, but the shape of the response function is very different from that of a linear system. The complex shape of $\mathrm{H}_{2}$ reveals the existence of a $1: 2: 2$ internal resonance which is already activated for this small amplitude of vibration. Two maxima are observed close to the two corresponding linear frequencies (denoted by the two dashed dotted lines $(-\cdot-))$. Finally, one can observe that the fourth harmonic $\left(H_{4}\right)$
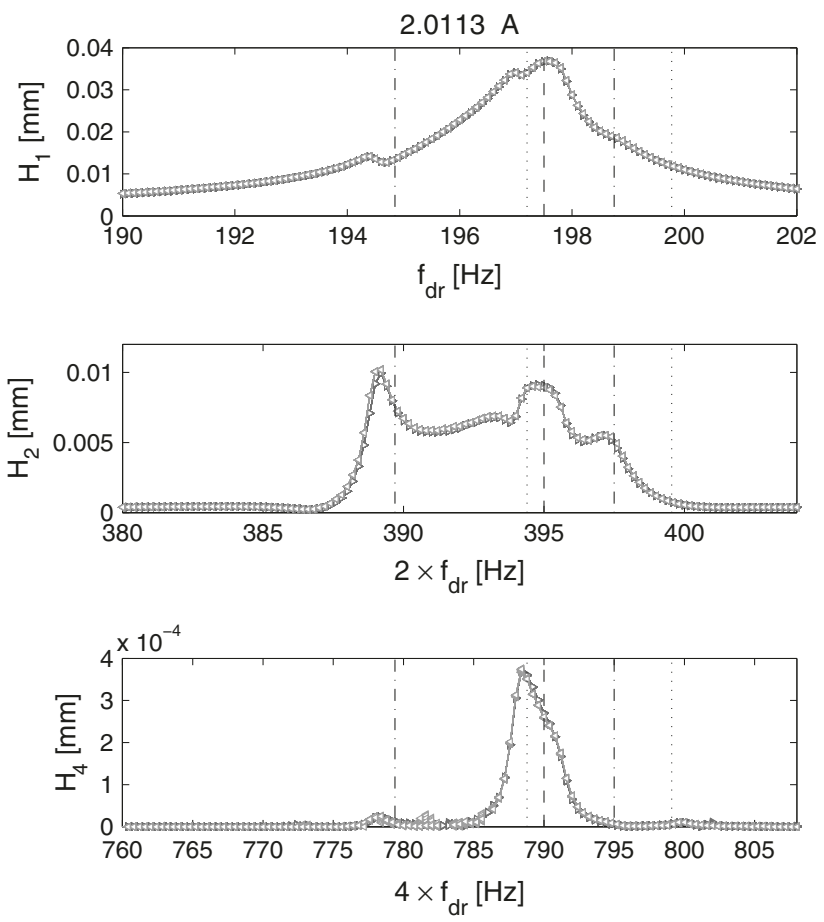

Fig. 9. Frequency response curves for the note G3 excited around the first mode $f_{d r} \simeq f_{1}=197.5 \mathrm{~Hz}$. Amplitudes of harmonic displacements for a weak amplitude of excitation $(I \simeq 2 A): H_{1} \simeq f_{d r}, H_{2} \simeq 2 f_{d r}$ and $H_{4} \simeq 4 f_{d r}$. Linear eigenfrequency markers $f_{1}(--), 2 f_{1}(-\cdot-)$ and $4 f_{1}(\cdots)$. Forward sweep $(-\triangleright-)$, backward sweep $(-\triangleleft-)$. 
presents a maximum at $4 f_{1}=788.8 \mathrm{~Hz}(\cdots)$, which is the identified eigenfrequency of one of the two degenerate modes shown in Fig. 6. The shape of those curves shares obvious similarities with the ones of the response of models with $1: 2,1: 2: 2$ or $1: 2: 4$ internal resonances $[20,25]$, that systematically show several peaks not precisely located at, but very close to, the resonance frequencies of the system. On the other hand, the second degenerate mode around $4 f_{1}$, whose eigenfrequency is $799.1 \mathrm{~Hz}$, is a bit too far from the main excited resonance so as to be activated by the nonlinear coupling. Forward and backward sweeps are completely superimposed so that for this level of excitation, no jump occurs in the system. This measurement clearly establishes that, for a very small level of vibration amplitude, 4 modes in 1:2:2:4 internal resonance interact and exchange energies in the system, resulting in a complex dynamics due to the intricated non linear behaviour.

A larger amplitude of excitation is now applied to the steelpan. Harmonic measurements are shown in Fig. 10, where the eighth harmonic is also shown. The dynamical response are now more complex and difficult to identify as compared to classical resonance curves of simple systems exhibiting, e.g. $1: 2: 2$ or $1: 2: 4$ internal resonance $[20,26,27]$. However, a peculiarity of the system's response is the occurence of jump phenomena and quasiperiodic responses, which are observed in the frequency band $[192.5,194] \mathrm{Hz}$. The participation of the second harmonic is the most important as its maximum value has been multiplied by a factor 8 as compared to the previous case for $I=2 \mathrm{~A}$. On the other hand the maximum amplitude of $H_{1}$ has increased of $50 \%$ only,
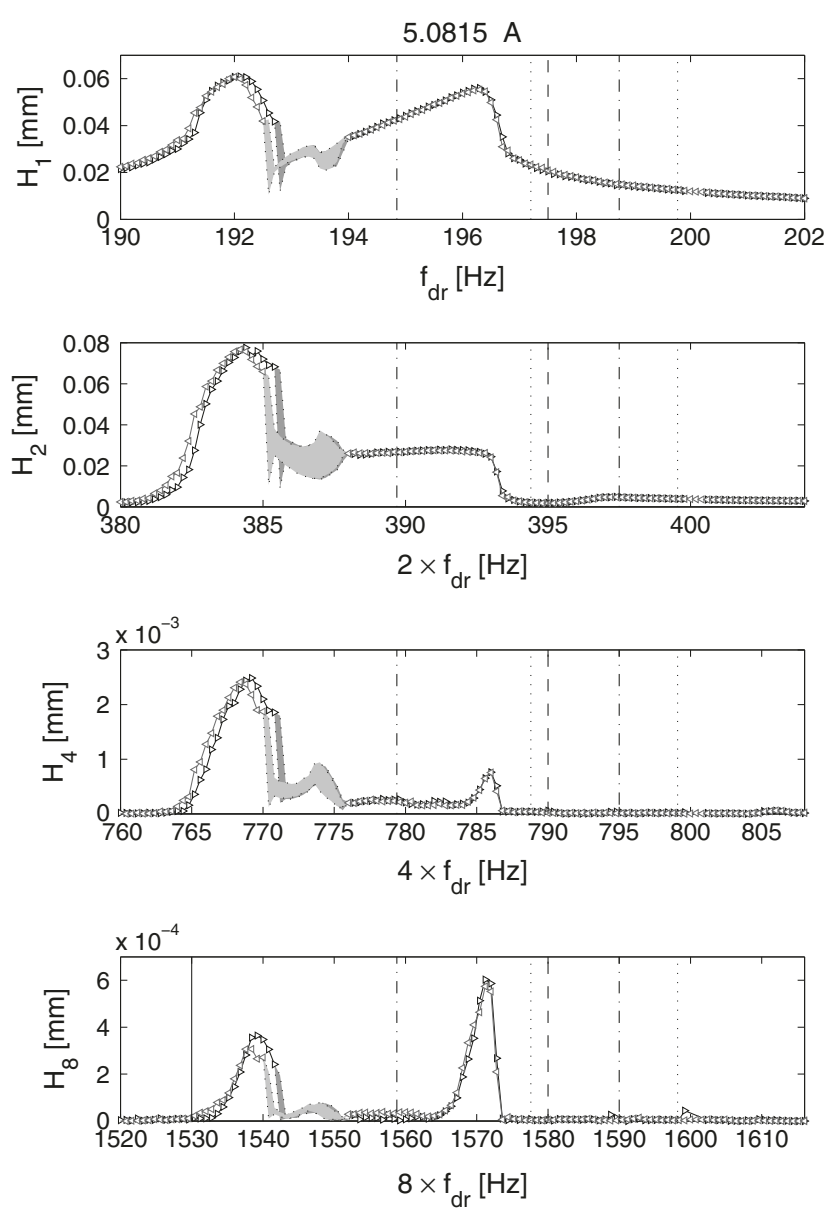

Fig. 10. Frequency response for the note $G 3$ excited around the first mode $f_{d r} \simeq f_{1}$. Amplitudes of harmonic displacements for a large amplitude of excitation $(I \simeq 5 A)$ : $H_{1}, H_{2}, H_{4}$ and $H_{8} \simeq 8 f_{d r}$. Linear eigenfrequency markers $f_{1}(--), 2 f_{1}(-\cdot-), 4 f_{1}(\cdots)$ and $8 f_{1}(-)$. Forward sweep $(-\triangleright-)$, backward sweep $(-\triangleleft-)$. which means that most of the injected energy, from those amplitude values, are almost directly transferred to the upper modes and more particularly to the second one. Transfer to the fourth harmonic is also very large so that the coupling identified for the smaller amplitudes still persist. Finally harmonic $H_{8}$ shows a significant contribution which means that a 1:2:2:4:8 coupling is surely excited. This measurement evidences the fact that the steelpan's eigenfrequencies are tuned so as to facilitate an easy transfer of energy from the low-frequency modes to the higher modes. This peculiarity has an important consequence on the sound produced by those instruments as they are capable of transferring rapidly energy up to the eigth harmonic.

\subsubsection{Mid-frequency excitation: $f_{d r} \simeq 2 f_{1}$}

In this section the frequency response curves for the G3 note of the steelpan are measured for an excitation frequency close to the first two degenerate modes, i.e. $f_{d r} \simeq 2 f_{1}$. As a coupling with the fundamental frequency mode is expected, and for unifying the notations and experimental procedures, $H_{2}$ still refers to the harmonic component in the vicinity of $f_{d r} \simeq 2 f_{1}$. Other harmonics are measured by the lock-in amplifier with a reference at half the driving frequency, in order to recover the subharmonic component at $f_{d r} / 2$, oscillating around the fundamental frequency. Two amplitudes of excitation are presented, a moderate value for which the current is $I \simeq 2 \mathrm{~A}$, and a strongly nonlinear one for which $I \simeq 5 \mathrm{~A}$.

Fig. 11 shows the measurement obtained for $I \simeq 2 \mathrm{~A}$, where 4 harmonics are shown. From the theoretical viewpoint, see e.g.
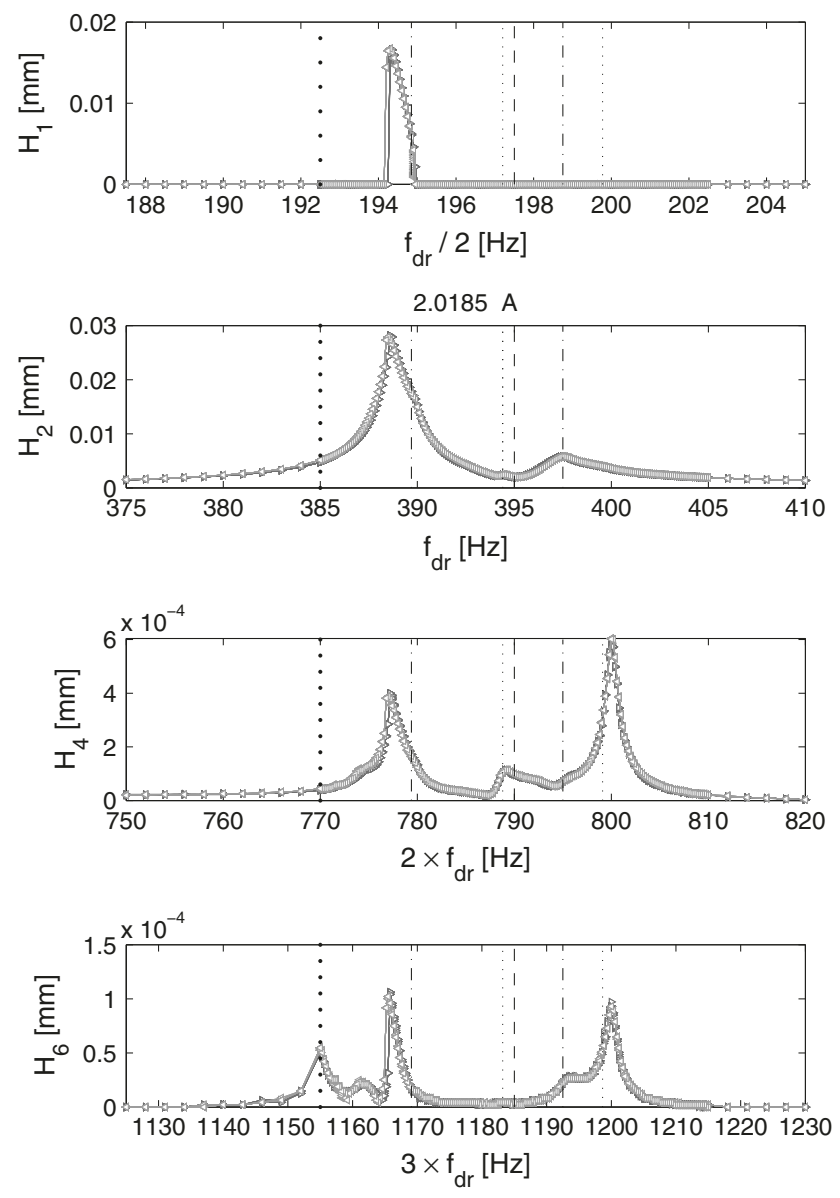

Fig. 11. Frequency response for the note $G 3$ excited with a mid-frequency excitation $F_{d} r \simeq 2 f_{1}$. Harmonic amplitude displacements for a weak amplitude of excitation $(I \simeq 2 A): H_{1} \simeq f_{d r} / 2, w H_{2} \simeq f_{d r}, H_{4} \simeq 2 f_{d r}$ and $H_{6} \simeq 3 f_{d r}$. Linear eigenfrequency markers $f_{1}(--), 2 f_{1}(-\cdot-), 4 f_{1}(\cdots)$ and $6 f_{1}(\cdots)$. Forward sweep $(-\triangleright-)$, backward sweep $(-\triangleleft-)$. 
$[20,26,27]$, one expects to obtain a direct (resonant) coupling between the degenerate modes at $2 f_{1}$ with those at $4 f_{1}$, with comparison to a $1: 2$ internal resonance excited on its lower frequency. Incidentally, it is observed that the mode at $389.7 \mathrm{~Hz}$ has a larger amplitude response than its companion at $397.5 \mathrm{~Hz}$. However, the direct coupling is effectively observed with a non-negligible response on the fourth harmonic. For the coupling with the fundamental (non-directly excited) mode, the theory states that an instability region exists and once the amplitude of $\mathrm{H}_{2}$ crosses this amplitude limit, then energy is transferred to the modes being at half the excitation frequency after a pitchfork bifurcation [20]. Here, this phenomenon is effectively measured, with a significant energy transfer to the fundamental (harmonic $H_{1}$ in Fig. 11) at half the excitation frequency $f_{d r}$, occurring on a narrow frequency interval $[194.2,194.8] \mathrm{Hz}$. Once again, this coupling, which needs a minimum amount of vibration response to occur, is here activated for a small excitation level, which means that the steelpan is tuned so as to favor such couplings.

Interestingly, the coupling scenario can be analyzed separately in the frequency band $f_{d r} \in[380 ; 393] \mathrm{Hz}$ (band 1), and $f_{d r} \in[393 ; 403] \mathrm{Hz}$ (band 2), because the degenerate modes eigenfrequencies are sufficiently well apart so as to interact individually. On the first frequency range $[380 ; 393] \mathrm{Hz}$, the $1: 2$ coupling with the fundamental mode is excited. Moreover, a non-negligible contribution of the sixth harmonic is observed, which let think that the mode at $1155 \mathrm{~Hz}$ is also excited. We can thus assume that a 1:2:6 coupling scenario is here present. On the second frequency range, [393;403] Hz, a clear 2:4:4 interaction is present between mode at $397.5 \mathrm{~Hz}$ and the two modes at 788.8 and $799.1 \mathrm{~Hz}$.

Fig. 12 shows the same case for a large excitation amplitude, $I \simeq 5 \mathrm{~A}$. As compared to the previous forcing amplitude, the following comments are worth mentionable:

- the global scheme identified before with the 1:2:6 resonance followed by the 2:4:4 is still present, and its characteristics are enhanced on a larger frequency range.

- a large hysteresis is now observed between forward and backward sweep around $f_{d r} \simeq 390 \mathrm{~Hz}$, with jump phenomena.

- a large coupling develops now between the fundamental mode the one at $389.7 \mathrm{~Hz}$. The second mode at $2 f_{1}$, with eigenfrequency $397.5 \mathrm{~Hz}$, still vibrates with a too small amplitude for exciting the fundamental one.

Once again, these measurements clearly establish the fact that, even for very small amplitudes of vibrations, numerous and complicated couplings between modes are present in the dynamical response of the steelpan. This undoubtedly emphasizes that energy exchanges are numerous in normal playing conditions, leading to complicated responses in the first milliseconds with efficient build-up of energy to the higher frequencies.

\subsection{Nonlinear response of the note F3}

The second experiment is realized by exciting the note F3 on the same right barrel of the double second steelpan. In this part, three cases of excitation are proposed: the driven frequency is applied in the low frequency case $\left(f_{d r} \simeq f_{1}\right)$, in the mid frequency case $f_{d r} \simeq 2 f_{1}$ and in the high frequency case $f_{d r} \simeq 4 f_{1}$. As in the previous sections, markers will be inserted into the figures for better readability and understanding of mode coupling. The markers are the same, with now the frequencies of the note F3, see Table 2.

\subsubsection{Low frequency excitation: $f_{d r} \simeq f_{1}$}

Fig. 13 shows the frequency response curves for three harmonics $\left(H_{1}, H_{2}\right.$ and $\left.H_{4}\right)$ when the F3 note is excited in the vicinity of its fundamental frequency, i.e. $f_{d r} \simeq 172 \mathrm{~Hz}$. The intensity in the
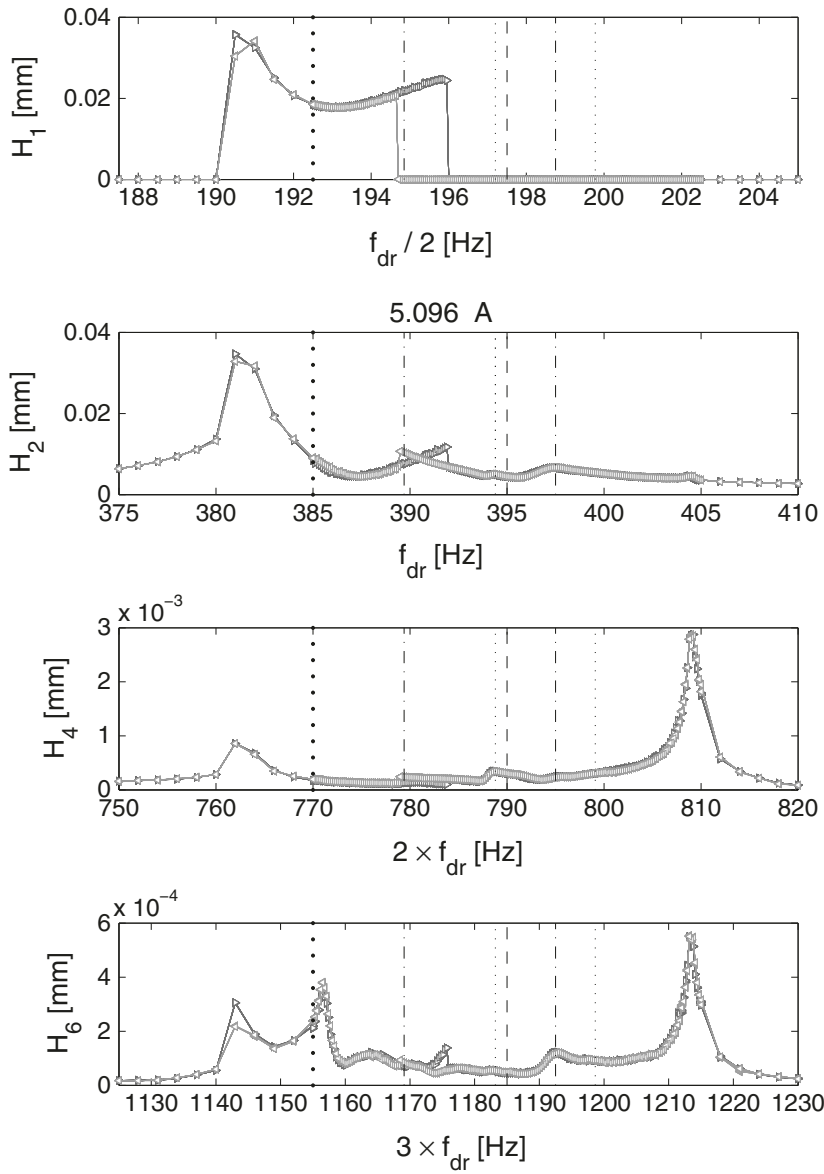

Fig. 12. Frequency response for the note G3 excited with a mid-frequency excitation $f_{d r} \simeq 2 f_{1}$. Harmonic amplitude displacements for a high amplitude of excitation $(I \simeq 5 A): H_{1} \simeq f_{d r} / 2, H_{2} \simeq f_{d r}, H_{4} \simeq 2 f_{d r}$ and $H_{6} \simeq 3 f_{d r}$. Linear eigenfrequency markers $f_{1}(--), 2 f_{1}(-\cdot-), 4 f_{1}(\cdots)$ and $6 f_{1}(\cdots)$. Forward sweep $(-\triangleright-)$, backward sweep $(-\triangleleft-)$.

Table 2

Eigenfrequencies identified for the note F3, and marker symbols used in all related figures (Figs. 13-18).

\begin{tabular}{lll}
\hline Frequency relationship & Value $(\mathrm{Hz})$ & Marker symbol \\
\hline$f_{1}$ & 172.5 & $(--)$ \\
$\simeq 2 f_{1}$ & 341.2 & $(-\cdot-)$ \\
& 357.5 & \\
$\simeq 4 f_{1}$ & 686.2 & $(\cdots)$ \\
& 705 & \\
\hline
\end{tabular}

current in this case is $I=0.42 \mathrm{~A}$, resulting in a vibration amplitude of the order of $0.04 \mathrm{~mm}$ for $H_{1}$. Note that this range of amplitude is of the order of the measurement for the note G3 (previous section), whereas the amplitude of the forcing is five times smaller. This is due to the fact that the coil/magnet system was located near the center of F3, whereas for G3 it was placed more on the periphery of the note (see the location of excitation points on Fig. 2). As expected from the linear resonance relationships and the theoretical results on 1:2:4 resonance [20], energy should be transferred easily up to the fourth harmonics. This feature is clearly observed, with a large response both on $\mathrm{H}_{2}$ and $H_{4}$, resulting in a complex shape for the first harmonic, far from a linear response, even though the vibration amplitude is less than one tenth the thickness.

Note that the detuning between the two degenerate modes at $2 f_{1}$ is large, such that the second mode at 357.5 is out of the coupling range. Hence a $1: 2: 2$ resonance is not possible in this case 

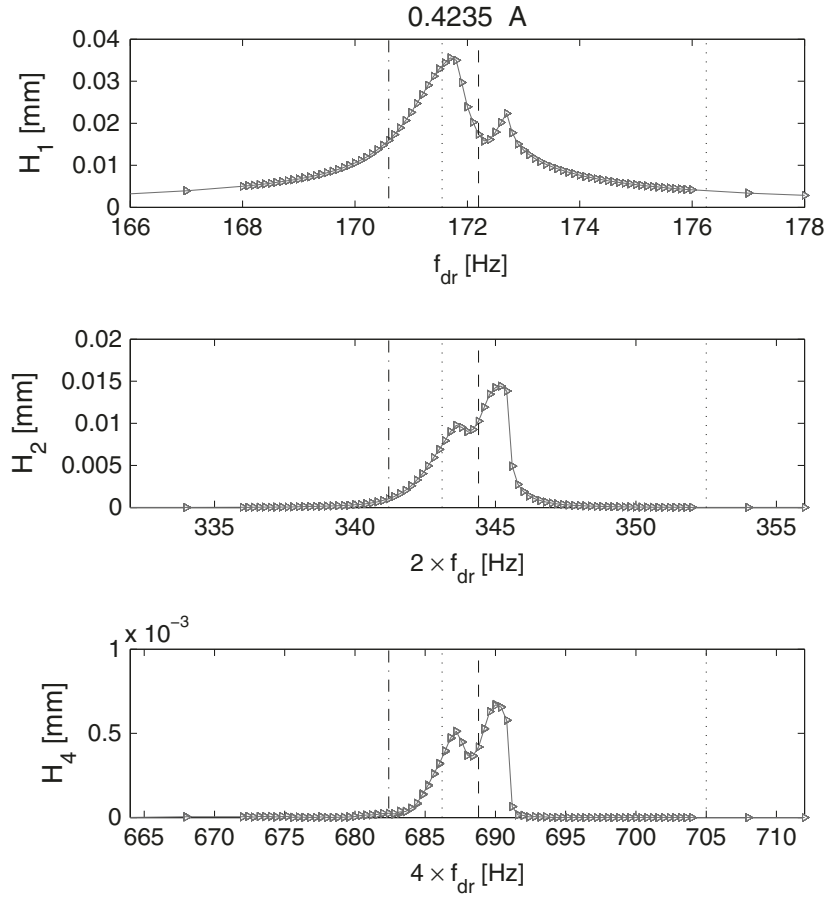

Fig. 13. Frequency response curve for the note $\mathrm{F} 3$ excited around the first mode $f_{d r} \simeq f_{1}$. Harmonic amplitude displacements for a small amplitude of excitation $(I \simeq 0.42 A): H_{1} \simeq f_{d r}, H_{2} \simeq 2 f_{d r}$ and $H_{4} \simeq 4 f_{d r}$. Linear eigenfrequency markers $f_{1}(--)$, $2 f_{1}(-\cdot-)$ and $4 f_{1}(\cdots)$. Forward sweep $(-\triangleright-)$.

and the system behaves (in terms of nonlinear couplings) as if only one mode is present at $2 f_{1}$. The same remark holds for the two modes at $4 f_{1}$, the upper at $705 \mathrm{~Hz}$ being obviously not excited. Hence a 1:2:4 internal resonance is here at hand.

Fig. 14 shows the measurements realized for a larger amplitude of excitation for which $I \simeq 2 \mathrm{~A}$. The behaviour is here strongly nonlinear with bent frequency response curves and vibration amplitudes of the order of two times the thickness, indicating that the cubic nonlinearities are also activated. A large jump phenomenon is observed between forward and backward excitation with a quasiperiodic regime around $f_{d r} \simeq 173.8 \mathrm{~Hz}$ on the forward sweep. For the backward sweep, the quasiperiodic regime is observed at $f_{d r} \simeq 172.6 \mathrm{~Hz}$. Following $H_{1}$ from the low frequencies, one observe first an increase of the response until a jump around $173.5 \mathrm{~Hz}$. However, the branch appearing after the jump is still strongly coupled to the higher modes and thus still in 1:2:4 internal resonance, with a saturation phenomenon for $\mathrm{H}_{2}$. This measurement shows that for vibration amplitudes of the order of the thickness, the steelpan displays complicated branch of solutions with strong activation of cubic nonlinearities.

\subsubsection{Mid frequency excitation: $f_{d r} \simeq 2 f_{1}$}

The F3 note is now excited in the vicinity of $2 f_{1}$, i.e. $f_{d r} \simeq 345 \mathrm{~Hz}$. A single amplitude of excitation, $I \simeq 0.6 \mathrm{~A}$, is presented in Fig. 15 with forward and backward sweep. The energy is simultaneously transferred from $\mathrm{H}_{2}$ to $\mathrm{H}_{1}$ and from $\mathrm{H}_{2}$ to $\mathrm{H}_{4}$, which means that a fully coupled case of a 1:2:4 scenario is once again involved. As in the low frequency excitation case, only one of each degenerate mode is activated, so that in that case, the complexity brought by the appearance of companion modes is not observed. A small jump is noticeable at $f_{d r} \simeq 345 \mathrm{~Hz}$ which mark a small difference between forward and backward sweeps.

\subsubsection{High frequency excitation: $f_{d r} \simeq 4 f_{1}$}

Finally, for the last experiment presented in this section, the driven frequency is around four times the fundamental, i.e.
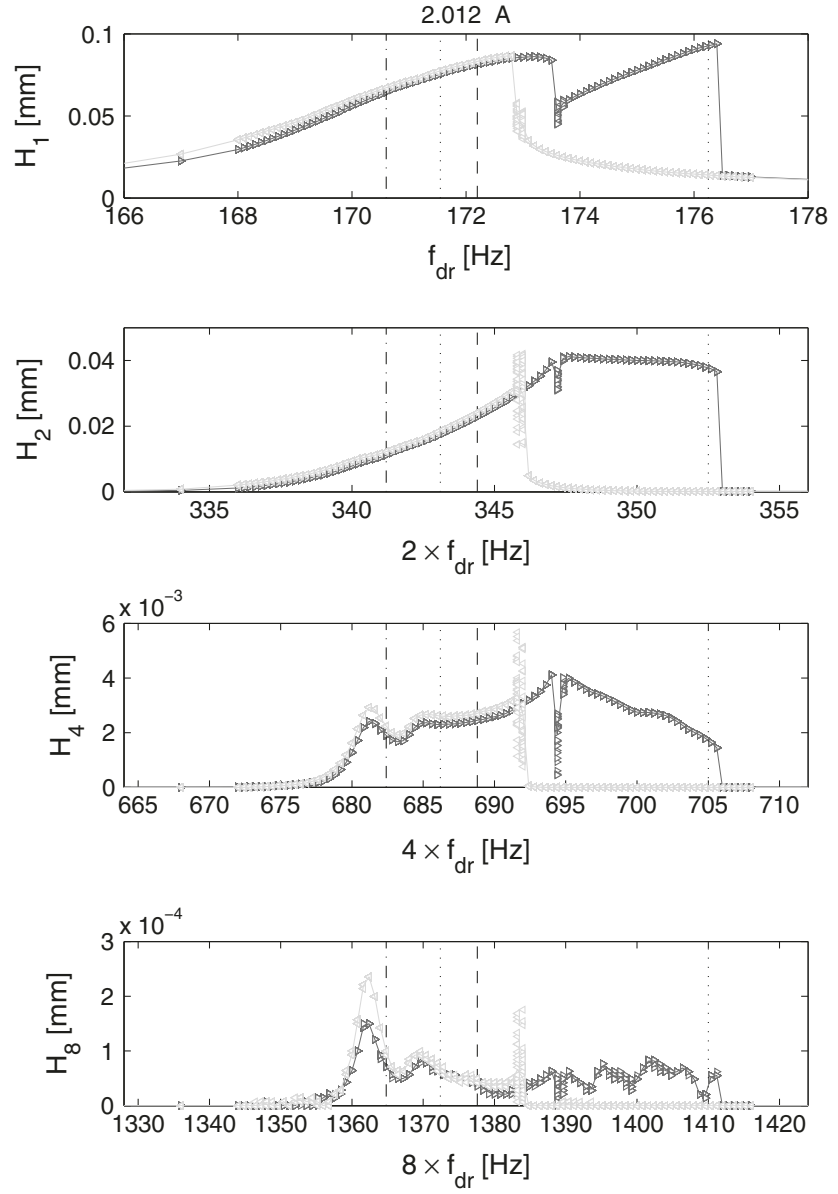

Fig. 14. Frequency response curve for the note $\mathrm{F} 3$ excited around the first mode $f_{d r} \simeq f_{1}$. Harmonic amplitude displacements for a high amplitude of excitation $(I \simeq 2 A): H_{1} \simeq f_{d r}, H_{2} \simeq 2 f_{d r}, H_{4} \simeq 4 f_{d r}$ and $H_{8} \simeq 8 f_{d r}$. Forward sweep $(-\triangleright-)$, backward sweep $(-\triangleleft-)$. Quasiperiodic oscillations are obtained for $f_{d r} \simeq 173 \mathrm{~Hz}$

$f_{d r} \simeq 680 \mathrm{~Hz}$. This case is particularly challenging since the goal is here to observe the cascade of coupling in a 1:2:4 resonance excited at its higher frequency, where energy can be transferred from $4 f$ to $2 f$ and then to $f[20]$. With that goal in mind, three amplitudes of excitations are shown to observe how the energy can be transferred to the lowest-frequency modes.

As already noted, the measurement time for each frequency response curve (of the order of $3 \mathrm{~h}$ ), which is very large, renders all the measurements difficult. Due to material problems, this last set of measurements have been realized one month after all the other ones, and the set-up has needed to be completely remounted. As a consequence of small displacements of sensors (in particular excitation magnet), a slight shift of some eigenfrequencies is expected. In order to measure correctly the eigenfrequency of one the two degenerate modes at $4 f_{1}$, a linear measurement is first performed and reported in Fig. 16, with a very small amplitude of excitation $(I \simeq 0.03 \mathrm{~A}$, resulting in vibration amplitude of $3.10^{-4} \mathrm{~mm}$ ). In that case the response is linear and the eigenfrequency is measured at $681 \mathrm{~Hz}$, instead of $686.2 \mathrm{~Hz}$ in the first series of measurements.

Fig. 17 shows a moderate amplitude of excitation, $I=1 \mathrm{~A}$, for which the directly excited harmonic $H_{4}$ is reported together with $\mathrm{H}_{2}$ and $\mathrm{H}_{8}$. The shape of $\mathrm{H}_{4}$ in the vicinity of the eigenfrequency is not linear anymore, and the shape of $H_{8}$ shows that a $1: 2$ resonance is already activated. More interestingly, the transfer of energy to the lower frequency mode at $2 f_{1}$ is observed on a small frequency range, where $\mathrm{H}_{2}$ departs from zero around $343 \mathrm{~Hz}$. We can then argue that a 2:4:8 internal resonance is observed. 

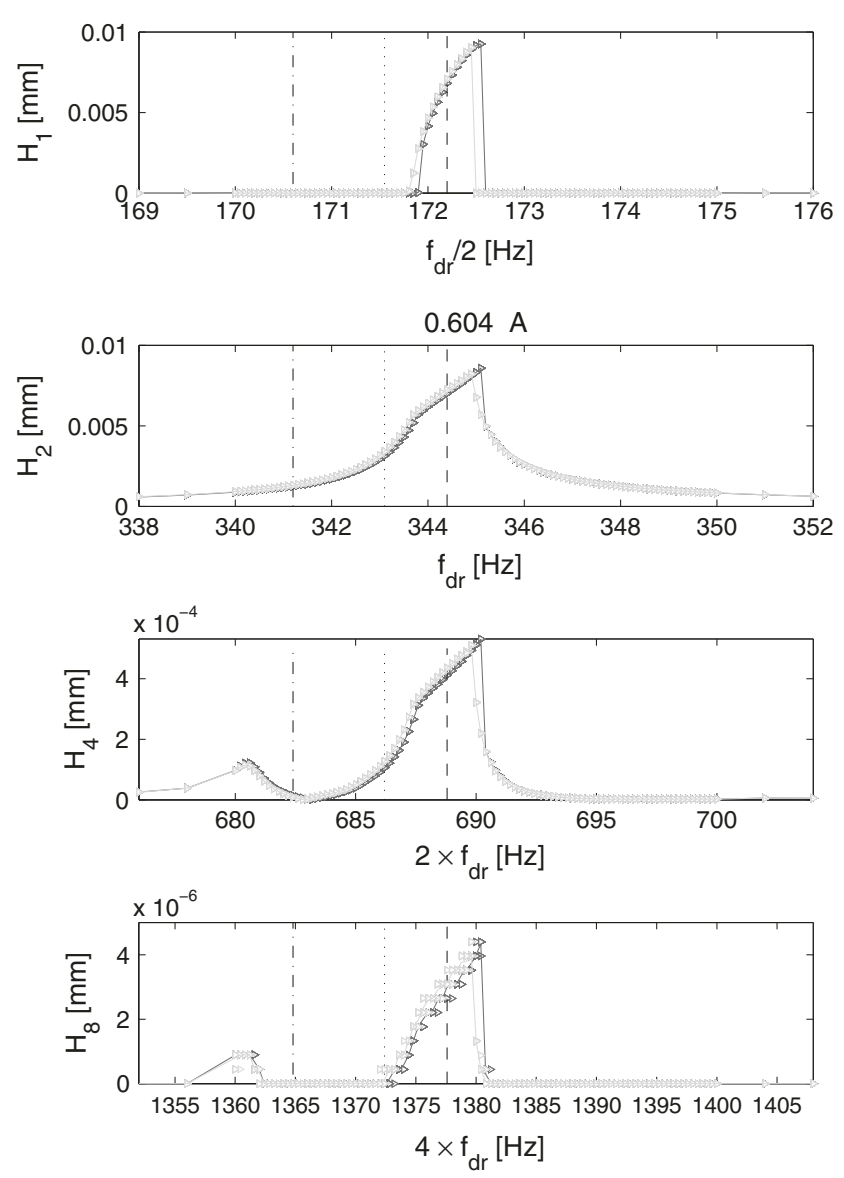

Fig. 15. Frequency response curve for the note $\mathrm{F} 3$ excited with a mid-frequency excitation $f_{d r} \simeq 2 f_{1}$. Harmonic amplitude displacements for $I \simeq 0.6 \mathrm{~A}: H_{1} \simeq f_{d r} / 2$,

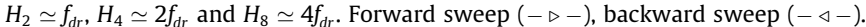

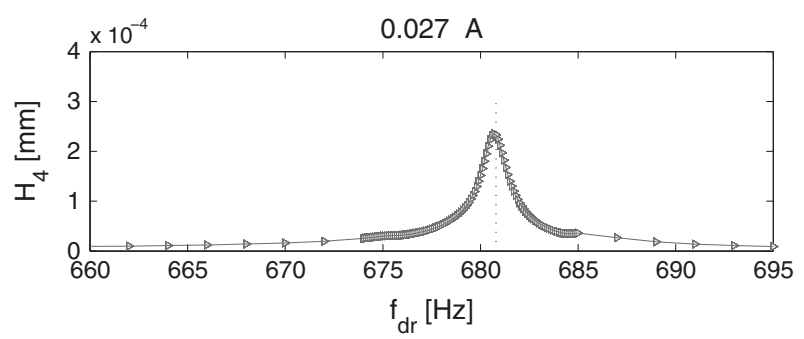

Fig. 16. Frequency response curve for the note $\mathrm{F} 3$ excited with a high-frequency excitation case $f_{d r} \simeq 4 f_{1}$. Harmonic amplitude displacements for $I \simeq 0.03 \mathrm{~A}: H_{4} \simeq f_{d r}$.

Finally, Fig. 18 shows the last measurement realized, for a large value of the forcing, $I=2 \mathrm{~A}$. In that case the transfer of energy down to the lowest frequency mode is observed, so that here a complete $1: 2: 4: 8$ resonance is measured. The behaviour is strongly nonlinear with two large frequency range on which quasiperiodic regimes are found. This observation is coherent with the theoretical results on the 1:2:4 resonance shown in [20], where it was emphasized that the presence of a third oscillator (as compared to a more classical $1: 2$ resonance) leads more easily to quasiperiodic regime. Here the complete coupling involves immediately the appearance of quasiperiodicity.

\subsection{Conclusion on the measurements}

The measurements made on the two notes of the doublesecond steelpan reveal a complex and rich dynamics featuring
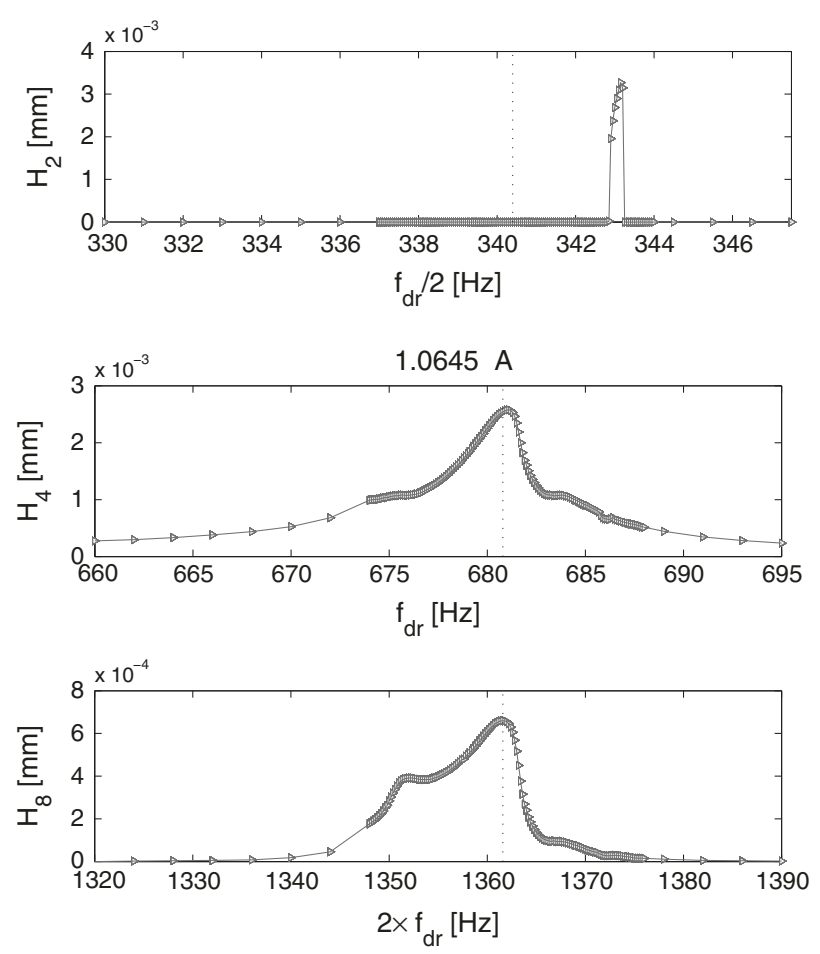

Fig. 17. Frequency-response curves for the note $\mathrm{F} 3$ excited with a high frequency $f_{d r} \simeq 4 f_{1}$. Harmonic amplitude displacements for $I \simeq 1 \mathrm{~A}: H_{2} \simeq f_{d r} / 2, H_{4} \simeq f_{d r}$ and $H_{8} \simeq 2 f_{d r}$.

numerous internal resonances and energy exchanges between internally resonant modes. Thanks to a harmonic, perfectly controlled excitation, we have been able to shed light on the most salient couplings appearing in permanent regime. In particular, the measurements clearly underline that $1: 2$ and $1: 2: 4$ internal resonance are excited for very small level of vibration amplitudes, and are at the ground basis of the dynamical solutions. This basic scheme is complexified by the fact that modes appear by pairs from the second one, so that the usual scheme is more that of a 1:2:2:4:4:6:8 dynamics. The presence of many modes favors unstable periodic states that have also been measured with the occurence of quasiperiodic regimes. Moreover, we have also observed that the couplings with modes at six or eight times the excitation frequency, are often excited, again complexifying the dynamical solutions. With that respect, the frequency response curves becomes very complicated as more and more modes are involved in the vibrations. Coming back to the normal playing where energy is inputted to the structure by an impact, one easily understands that these couplings, being excited for very small vibration amplitudes, are present and are the key to understand the peculiar sound of the steelpan with the rapid build-up of energy to higher modes.

The objective of the remainder of the paper is twofold. First to identify the values of some of the quadratic nonlinear coupling terms. For that, two simple models displaying respectively $1: 2: 2$ and 1:2:4 internal resonances are considered, since they provide analytical solutions [20], for a reasonable complexity. Higher-order models with more modes involved would be too difficult to fit on the measurements because of the number of coefficients to fit. Once these values identified, we will show that a 1:2:4 model with all the fitted parameters allows to recover the main features of the dynamical solutions of an impacted note, hence definitely showing that these internal resonances are key for a meaningful understanding of steelpan's vibrations. 

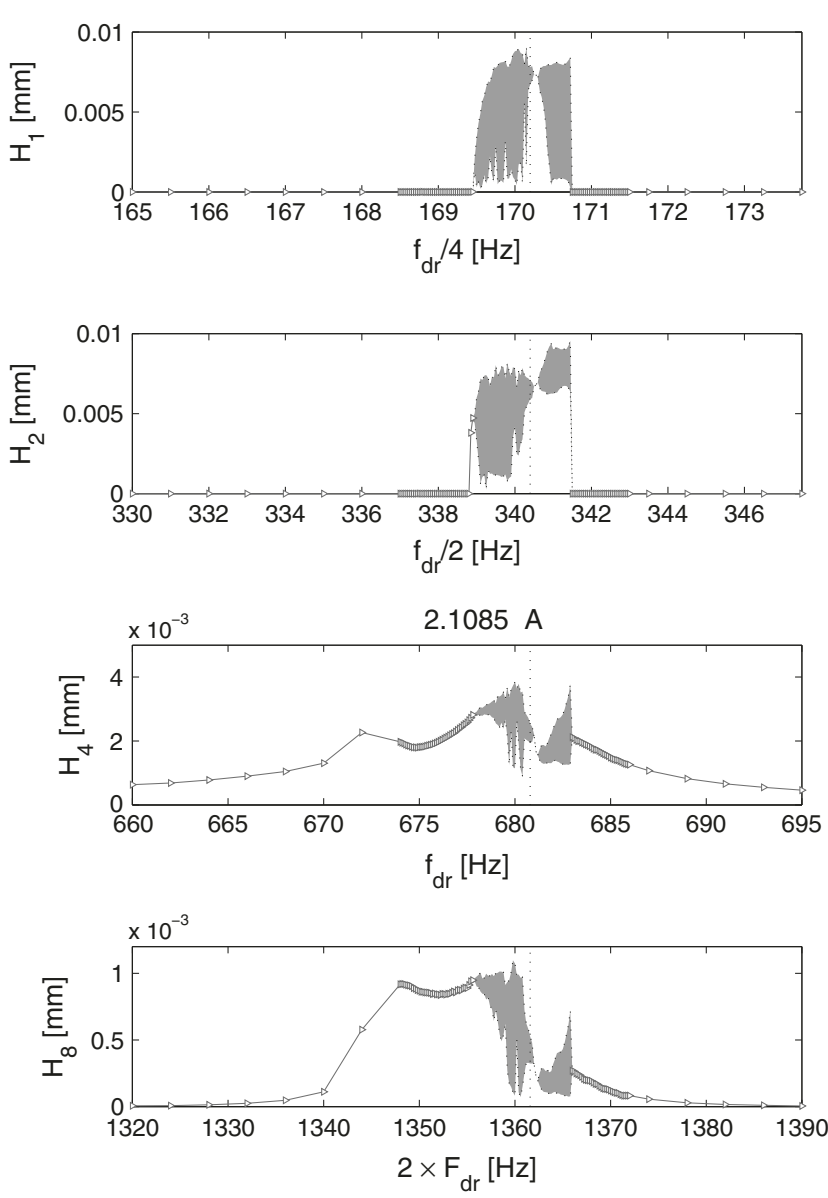

Fig. 18. Frequency-response curves for the note $\mathrm{F} 3$ excited with a high frequency $f_{d r} \simeq 4 f_{1}$. Harmonic amplitude displacements for $I \simeq 2 \mathrm{~A}: H_{1} \simeq f_{d r} / 4, H_{2} \simeq f_{d r} / 2$, $H_{4} \simeq f_{d r}$ and $H_{8} \simeq 2 f_{d r}$. The gray regions depict quasiperiodic oscillations.

\section{Theoretical models - nonlinear parameter identification}

\subsection{Model equations}

\subsubsection{The 1:2:2 internal resonance model}

The 1:2:2 internal resonance model displays three modes which eigenfrequencies are tuned such that $\omega_{3} \simeq \omega_{2} \simeq 2 \omega_{1}$. The nonlinear dynamics, considering quadratic nonlinear terms due to geometric nonlinearity only, reads [20]:

$$
\begin{aligned}
& \ddot{q}_{1}+\omega_{1}^{2} q_{1}=\left[-2 \xi_{1} \omega_{1} \dot{q}_{1}-\alpha_{1} q_{1} q_{2}-\alpha_{2} q_{1} q_{3}+F_{1} \cos (\Omega t)\right], \\
& \ddot{q}_{2}+\omega_{2}^{2} q_{2}=\left[-2 \xi_{2} \omega_{2} \dot{q}_{2}-\alpha_{3} q_{1}^{2}\right], \\
& \ddot{q}_{3}+\omega_{3}^{2} q_{3}=\left[-2 \xi_{3} \omega_{3} \dot{q}_{3}-\alpha_{4} q_{1}^{2}\right],
\end{aligned}
$$

where $\omega_{k}=2 \pi f_{k}$ is the angular frequency of mode $k$ and $\xi_{k}$ is the associated modal damping ratio. Only the case of a forcing in the vicinity of the first eigenfrequency is considered here, so that the excitation is on the first oscillator only, with an amplitude $F_{1}$ and a driving frequency $f_{d r}=\Omega / 2 \pi$, where $\Omega \simeq \omega_{1}$. The theoretical response, described in details in [20], can be interpreted as two nested well-known 1:2 internal resonances [25,27,28].

When the system is driven by $\Omega \simeq \omega_{1}$, the first-order solutions of Eqs. (1) are obtained by the multiple scales method as [20]:

$$
\begin{aligned}
& q_{1}(t)=a_{1} \cos \left(\Omega t+\varphi_{1}\right), \\
& q_{2}(t)=a_{2} \cos \left(2 \Omega t+\varphi_{2}\right), \\
& q_{3}(t)=a_{3} \cos \left(2 \Omega t+\varphi_{3}\right),
\end{aligned}
$$

where $a_{k}$ and $\varphi_{k}$ are amplitudes and phases of the solutions $q_{k}$, respectively. One can note that driving the system on its low-frequency mode creates two upper harmonic solutions, oscillating at twice the driven frequency.

In order to properly compare the measurements realized at a given point of the structure with the theoretical model, one has first to reconstruct the transverse displacement $w(t)$ at a given point of the steel-pan upper surface as:

$w(t)=w_{1} \cos \left(\Omega t+\varphi_{1}\right)+w_{2} \cos \left(2 \Omega t+\psi_{2}\right)$.

Whereas $w_{1}$ is easily related to $a_{1}$ with the formula $w_{1}=a_{1} \Phi_{1}$ (where $\Phi_{1}$ refers to the modal amplitude of mode 1 at the measurement point); $w_{2}$ and $\psi_{2}$ involve combinations of the amplitude solutions $a_{2}, a_{3}$, phases $\varphi_{2}, \varphi_{3}$ as well as modal amplitudes $\Phi_{2}, \Phi_{3}$ at the measurement point. The detailed calculation is given in Appendix A. Finally, Eq. (3) allows to compute, from the model, the two harmonics amplitudes $\mathrm{H}_{1}$ and $\mathrm{H}_{2}$ of the displacement at the measurement point, so as to compare them with the measurements.

\subsubsection{The 1:2:4 internal resonance model}

The second model proposed here is a 1:2:4 internal resonance model. Three modes oscillating at $\omega_{3} \simeq 2 \omega_{2} \simeq 4 \omega_{1}$ are now considered. The system equations reads [20]:

$$
\begin{aligned}
& \ddot{q}_{1}+\omega_{1}^{2} q_{1}=\left[-2 \xi_{1} \omega_{1} \dot{q}_{1}-\alpha_{5} q_{1} q_{2}+F_{1} \cos \Omega t\right], \\
& \ddot{q}_{2}+\omega_{2}^{2} q_{2}=\left[-2 \xi_{2} \omega_{2} \dot{q}_{2}-\alpha_{6} q_{1}^{2}-\alpha_{7} q_{2} q_{3}\right], \\
& \ddot{q}_{3}+\omega_{3}^{2} q_{3}=\left[-2 \xi_{3} \omega_{3} \dot{q}_{3}-\alpha_{8} q_{2}^{2}\right] .
\end{aligned}
$$

Compared to the $1: 2: 2$ case, four different resonant coupling coefficients $\alpha_{i}$ are introduced. Once again, the forcing is considered on the first oscillator as we restrict ourselves to an excitation frequency in the vicinity of the first eigenfrequency.

With this model three amplitude solutions are obtained as:

$$
\begin{aligned}
& q_{1}(t)=a_{1} \cos \left(\Omega t+\varphi_{1}\right), \\
& q_{2}(t)=a_{2} \cos \left(2 \Omega t+\varphi_{2}\right), \\
& q_{3}(t)=a_{3} \cos \left(4 \Omega t+\varphi_{3}\right) .
\end{aligned}
$$

The reconstruction of the transverse displacement $w$ is simpler than in the 1:2:2 case, because only one amplitude solution per oscillating frequency is present. Hence, when $\Omega \simeq \omega_{1}$, the transverse displacement at the measurement point writes:

$w(t)=w_{1} \cos \left(\Omega+\varphi_{1}\right)+w_{2} \cos \left(2 \Omega+\varphi_{2}\right)+w_{3} \cos \left(4 \Omega+\varphi_{3}\right)$,

where $w_{1}=a_{1} \Phi_{1}, w_{2}=a_{2} \Phi_{2}$ and $w_{3}=a_{3} \Phi_{3}$, and $\Phi_{p}$ corresponding to the amplitude of the eigenmode at the measurement point.

\subsection{Model fitting}

The fitting procedure consists in adjusting all the model parameters so that the theoretical resonance curves fit the experimental ones. The values of the natural frequencies $\omega_{p}$ and the modal damping ratio $\xi_{p}$ are identified from a fit (not shown here) of the linear response of the model on resonance curves with a very low driving amplitude. The mode shapes $\Phi_{p}$ are obtained from the operational deflection shapes of Fig. 6 and 7, measured with a laser vibrometer. They are normalized so that $\Phi_{p}=1 \forall p$ at the excitation point. Then, the values of the nonlinear coupling coefficients $\alpha_{i}$, the main unknowns, are identified on the nonlinear resonant response of the steelpan.

As compared to other similar works of the literature (e.g. [22] for $1: 1$ internal resonance in circular plates and [23] for $1: 1: 2$ internal resonance in spherical shells), the present case is rendered difficult because for all the measured nonlinear response curves, always more than three modes are resonantly coupled, so that both three degree of freedom models are not as realistic as they should be. For those reasons, only a few fits are reported here: one from 
the G3 note and one from the F3 note, both excited at a small level and in the vicinity of the fundamental frequency.

Models (1) and (4) are dimensionless, so as the fitted values of the $\alpha_{i}$ and of the forcing amplitude $F_{1}$. These dimensionless variables (denoted here with overbars) are defined with the following amplitude and time scalings:

$\bar{w}=\frac{w}{w_{0}}, \quad \bar{\Omega}=\frac{\Omega}{2 \pi f_{0}}, \quad \bar{t}=2 \pi f_{0} t$

with $w_{0}=1 \mathrm{~mm}, f_{0}=197 \mathrm{~Hz}$ for the G3 note and $f_{0}=171.7 \mathrm{~Hz}$ for the F3 note.

\subsubsection{Second G3: low-frequency excitation/1:2:2-1:2:4 internal resonance models}

The first case corresponds to the measurement shown in Fig. 9, for which a clear 1:2:2:4 internal resonance scenario has been identified in Section 3.2.1. As a complete model involving 1:2:2:4 have no simple analytical solutions and would display too many free parameters for the fitting, we show the results obtained by both the 1:2:2 and the 1:2:4 models, in order to show their ability to retrieve the most important parts of the frequency response curves for harmonics $\mathrm{H}_{1}, \mathrm{H}_{2}$ and $\mathrm{H}_{4}$.

The linear parameters of the models (Eqs. (1)), namely the eigenfrequencies $f_{k}$, the modal damping ratios $\xi_{k}$ and the modal amplitudes at measurement points $\Phi_{k}$, are retrieved from the linear modal analysis. Variations of these parameters have been allowed in order to obtain the best possible fit. This is justified by the fact that linear parameters are very sensitive to experimental variations (e.g. temperature, position of magnet), that can vary from one experiment to the other. We recall that the measurement of a given nonlinear response curve lasts about three hours (see Section 3.1). The nonlinear coupling parameters $\alpha_{k}$ of the two models are left completely free and selected so as to obtain the best fit.

The results of the two fits are shown in Fig. 19. The linear parameters are given in Tables 3 and 4. One can observe that variations have been allowed in order to obtain the best fit. For the 1:2:2 model, the following values of the nonlinear coupling terms have been found to give the best frequency response curves: $\alpha_{1}=0.42, \alpha_{2}=0.46, \alpha_{3}=0.46, \alpha_{4}=0.39$, with $F_{1}=6.3 \cdot 10^{-4}$. One can observe that the 1:2:2 model allows to recover with a good accuracy the two peaks in the second harmonic (component $w_{2}$ ), and gives a correct approximation of the frequency response of the first harmonic (component $w_{1}$ ) before the fundamental frequency. However, as expected from the model, the oscillator at $4 f_{1}$ is missing which result in a poor approximation of the peaks appearing in the three measured harmonics around $197 \mathrm{~Hz}$. The missing information is retrieved when considering the 1:2:4 model, which allows to recover the main peak of the first harmonic, the peak on $w_{2}$ around $394 \mathrm{~Hz}$ and the resonance of the third mode at $788 \mathrm{~Hz}$ on component $w_{3}$. The nonlinear coefficients for the 1:2:4 model have been found to be equal to: $\alpha_{5}=0.24, \alpha_{6}=0.34, \alpha_{7}=0.88, \alpha_{8}=0.53$, with $F_{1}=12 \cdot 10^{-4}$. One can observe that all the nonlinear coefficients have the same order of magnitude, indicating the strength of the quadratic nonlinearity.

These fits definitely ensure that a $1: 2: 2: 4$ resonance scenario is here activated, and allows us to obtain values of the nonlinear coupling coefficients that can be used to feed a time model including the correct geometric nonlinearities, proposed in Section 5.

\subsubsection{Second F3: low-frequency excitation/1:2:4 internal resonance} model

The second selected example is the low-frequency excitation of the note F3 shown in Fig. 13. In that case, a 1:2:4 scenario has been identified in Section 3.3, and the occurence of a more complex dynamical solution involving the companion mode of either the
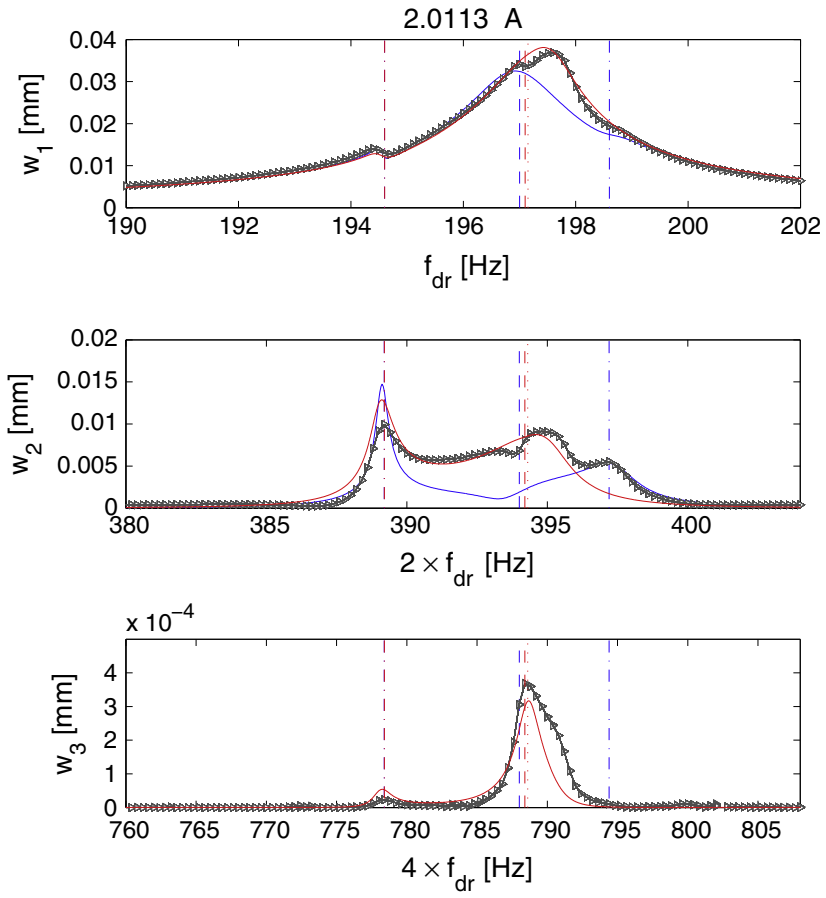

Fig. 19. Model fitting to experiments for the $\mathrm{G} 3$ note excited at moderate amplitude $(I=2 \mathrm{~A})$. Frequency response curves for harmonics $H_{1}\left(w_{1}\right), H_{2}\left(w_{2}\right)$ and $H_{4}\left(w_{3}\right)$. Measurements (black line and triangles $\triangleright$ ) versus 1:2:2 model (blue solid line) with $\alpha_{1}=0.42, \alpha_{2}=0.46, \alpha_{3}=0.46, \alpha_{4}=0.39$, and 1:2:4 model (red solid line) with $\alpha_{5}=0.24, \alpha_{6}=0.34, \alpha_{7}=0.88, \alpha_{8}=0.53$. (For interpretation of the references to color in this figure legend, the reader is referred to the web version of this article.)

Table 3

Linear parameters used for the fitting of the 1:2:2 model of Eqs. (1), shown in Fig. 19

\begin{tabular}{llll}
\hline & Osc. 1 & Osc. 2 & Osc. 3 \\
\hline$f_{k}(\mathrm{~Hz})$ & 197.002 & 389.2 & 397.2 \\
$\xi_{k}$ & 0.0051 & 0.0005 & 0.0023 \\
$\Phi_{k}$ & 0.56 & 0.47 & 0.53 \\
\hline
\end{tabular}

Table 4

Linear parameters used for the fitting of the 1:2:4 model of Eqs. (4), shown in Fig. 19

\begin{tabular}{llll} 
& Osc. 1 & Osc. 2 & Osc. 3 \\
\hline$f_{k}(\mathrm{~Hz})$ & 197.1 & 389.2 & 788.6 \\
$\xi_{k}$ & 0.0045 & 0.001 & 0.001 \\
$\Phi_{k}$ & 0.30 & 0.34 & 0.06 \\
\hline
\end{tabular}

mode at $2 f_{1}$ or the one at $4 f_{1}$ has been discarded since the detuning was too large between the eigenfrequencies. The fitting procedure is the same as in the previous section: the linear parameters are derived from the modal analysis and variations are allowed. The values used for the fit are given in Table 5 . The nonlinear coefficients have been identified as: $\alpha_{5}=0.48, \alpha_{6}=0.57, \alpha_{7}=0.68$ $\alpha_{8}=0.43$, with $F_{1}=2.13 \cdot 10^{-4}$. They are of the same order of magnitude as those for the note G3. The result of the fitting is shown in Fig. 20. One can note that most of the important parts of the frequency response curves are correctly reproduced by the model. However, a detail in the main resonance is still missing: the experimental frequency peak at resonance around $172.8 \mathrm{~Hz}$ is still underestimated by the model, and the secondary peak on the second harmonic ( $w_{2}$ at $343 \mathrm{~Hz}$ ) and more obviously on the fourth harmonic $\left(w_{3}\right.$ at $\left.687 \mathrm{~Hz}\right)$ is not large enough in the model. An assumption would be that another coupling, for example with the mode at $8 f_{1}$, is already activated, so that a $1: 2: 4: 8$ scenario is already involved in the measurement. However, $\mathrm{H}_{8}$ has not been measured in that case so that we are led to conclude that the 
Table 5

Linear parameters used for the fitting of the 1:2:4 model of Eqs. (4), shown in Fig. 20.

\begin{tabular}{llll}
\hline & Osc. 1 & Osc. 2 & Osc. 3 \\
\hline$f_{k}(\mathrm{~Hz})$ & 171.7 & 344.8 & 689.7 \\
$\xi_{k}$ & 0.003 & 0.0006 & 0.0013 \\
$\Phi_{k}$ & 1.002 & 1.06 & 0.95 \\
\hline
\end{tabular}

$1: 2: 4$ resonance is able to catch the most important part of the dynamics in this case, but a more complicated resonance should already be activated so that the frequency responses still present a small discrepancy with the theoretical one.

As a conclusion on this section, simple 3-dofs models displaying $1: 2: 2$ and $1: 2: 4$ internal resonance have been used to fit experimental frequency response curves. In the two tested cases, good results have been found, allowing to:

- obtain a numerical estimate for the nonlinear quadratic coupling coefficient,

- better qualify the resonance scenario involved in some frequency response curve.

In particular, it has been shown that even in a simple case as the 1:2:4 scenario found for the F3 note, some details of the frequency-response curve may be missed by the model, indicating that a more complex dynamics may be already activated. In the other experimental cases shown in Section 3 for larger amplitudes of excitation, the fits have not been made possible since the scenario are more complex, with more modes involved, as well as with an evident participation of cubic nonlinearities, not taken into account in the simple models. However, the proposed fittings allowed to retrieve an estimate of the quadratic coupling terms as well as an assessment of the 1:2:4 scenario as key to drive the nonlinear response in steelpan's vibrations. This will be further illustrated in the last section of the paper, by considering free (impacted) vibrations, and showing that the simple fitted model
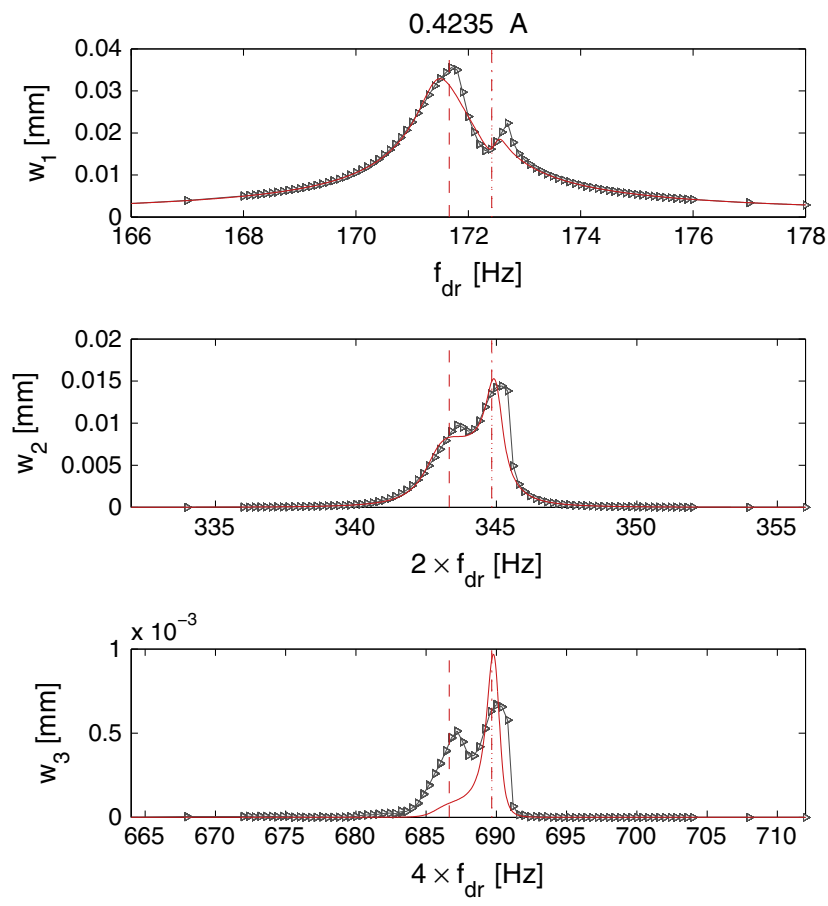

Fig. 20. Model fitting to experiments for the $\mathrm{F} 3$ note excited at moderate amplitude $(I=0.42 \mathrm{~A})$. Frequency response curves for harmonics $H_{1}\left(w_{1}\right), H_{2}\left(w_{2}\right)$ and $H_{4}\left(w_{3}\right)$. Measurements (black line and triangles $\triangleright$ ) versus 1:2:4 model (red solid line) with $\alpha_{5}=0.48, \alpha_{6}=0.57, \alpha_{7}=0.68, \alpha_{8}=0.43$. (For interpretation of the references to color in this figure legend, the reader is referred to the web version of this article.) allows recovering the time variations of the first four harmonics of the signal.

\section{Free oscillations}

The objective is to compare the time domain response of an impacted note of the steelpan in usual playing with the time response of a simple model exhibiting 1:2:4 internal resonance for the parameters identified in the previous section. The measurement is presented first.

\subsection{Experiment in free oscillations}

The time domain acceleration of a single point of the vibrating G3 note is considered, in usual condition of playing. An accelerometer, glued at a given position in the vicinity of the center of the G3 dome, is used. A musician hits the note as usual and for two different impact forces, roughly corresponding to a mezzo-piano and a forte intensity. The spectrograms of the two time acceleration signals are shown in Fig. 21. Whereas the energy is mainly concentrated into the two first components for the weak strike, Fig. 21(a), a strong build-up of energy to higher modes is evident in the strong strike, with significant energy levels up to $1700 \mathrm{~Hz}$. This particular behaviour is typical of the fact that the geometric nonlinearity is excited in the second case, so that energy exchanges are possible thanks to internal resonances scenario, and particularly to the $1: 2: 4$ resonance. In the weak strike where the vibration is mostly linear, the energy is confined to the modes where it has been inputted by the impact.

\subsection{Filtered time response}

In order to more precisely follow the time evolution of the first four harmonics $\mathrm{H}_{1}, \mathrm{H}_{2}$ and $\mathrm{H}_{4}$, a filter based on a short-time Fourier transform procedure, and allowing to extract the time domain
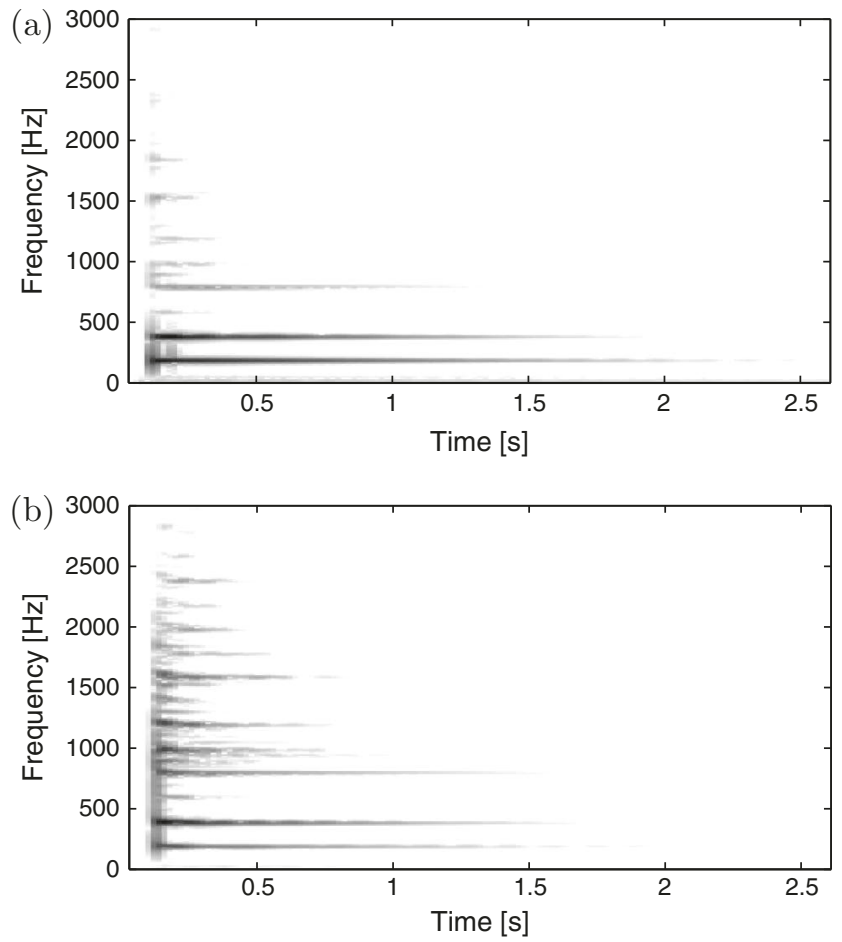

Fig. 21. Experimental spectrograms of free oscillations measurements with two stick-impact intensities on note G3 of the double second. (a) Weak strike (mezzopiano). (b) Strong strike (forte). 
response of these partials, is used. The three first frequency components (oscillating at $f_{1}, 2 f_{1}$ and $4 f_{1}$ ) are extracted from each measurement, are shown in Fig. 22. For the mezzo-piano intensity, Fig. 22(a), most of the energy is concentrated into $H_{1}$ and $H_{2}$. The nonlinear coupling between the two components is already present as attested by the small time delay between the maximum amplitudes of $H_{1}$ and $H_{2}$, clearly assessing that part of the energy directly transferred into $H_{1}$ by the impact is then transmitted by nonlinear couplings to $\mathrm{H}_{2}$. For the forte intensity, Fig. 22(b), the transfer of energy is so evident that $\mathrm{H}_{2}$ reaches an amplitude level of four times $H_{1}$, and $H_{4}$ strongly participates to the vibration with an amplitude comparable to that of $H_{1}$. Finally, one can also observe modulation in the time response for the harmonics (more particularly pronounced for $\mathrm{H}_{2}$ ), with a period decreasing with the strike amplitude. This is again an obvious manifestation of a nonlinear behaviour, with the energy coming back and forth to each oscillators through the nonlinear coupling terms (see e.g. [25] for a general two degree of freedom model with quadratic nonlinearities and $[2-4]$ in the case of steelpans).

\subsection{Simulations with the 1:2:4 model}

The 1:2:4 internal resonance model presented in Section 4.1 .2 is used to recover the behaviour of the first four harmonics shown in Fig. 22. The model is used with the parameters identified from the experiment in forced vibrations, and given in Section 4.2.1. The model is integrated in time domain using a fourth-order RungeKutta scheme with a variable timestep (routine "ode45" built in Matlab) [29]. An initial condition, imposed on the first oscillator only (at $t=0, w_{1}=w_{10}, w_{2}=w_{3}=0$ in Eq. (6)), with a selected amplitude in displacement, is used so as to mimic the impact brought by the stick hammer. Two different amplitudes of initial conditions are used for reproducing the impacts: $w_{10}=0.15 \mathrm{~mm}$
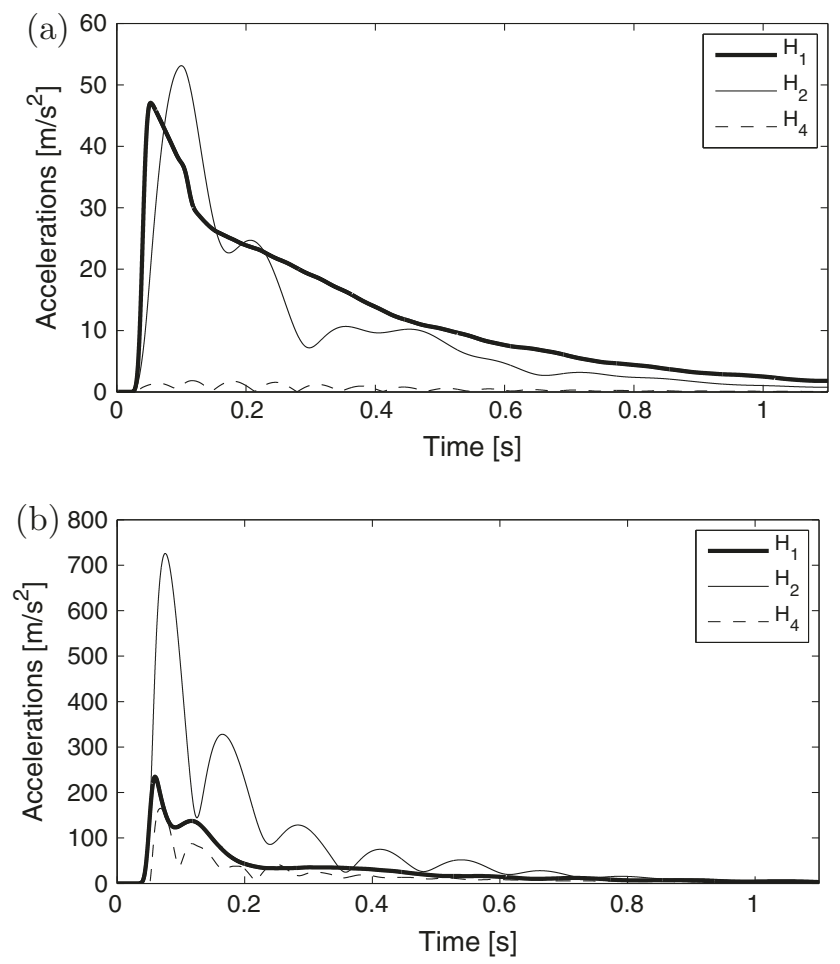

Fig. 22. Time domain evolution of harmonics $H_{1}, H_{2}$ and $H_{4}$ extracted from the time response of the signals which spectrograms are shown in Fig. 21. (a) Weak strike (mezzo-piano). (b) Strong strike (forte).
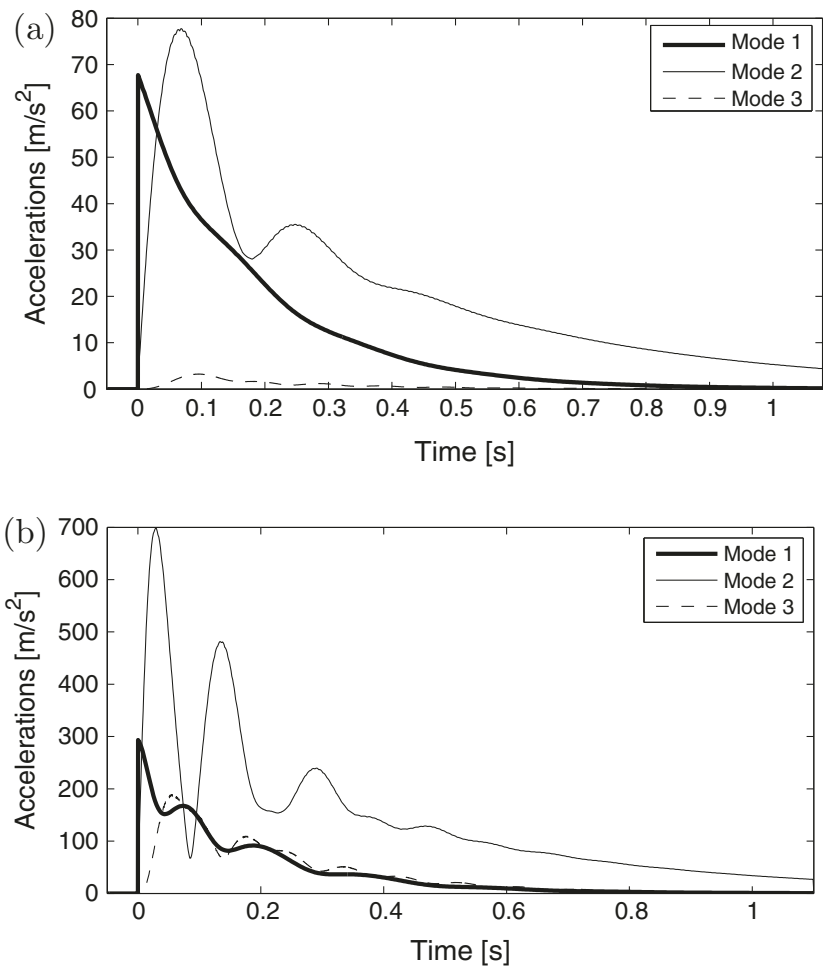

Fig. 23. Time domain evolution of harmonics $w_{1}, w_{2}$ and $w_{3}$ extracted from the time response of the model presenting a 1:2:4 internal resonance, which coefficients have been adjusted from the forced frequency response curves. Initial conditions in displacement: (a) $w_{10}=0.15 \mathrm{~mm}$, (b) $w_{10}=0.65 \mathrm{~mm}$.

for the mezzo-piano one and $w_{10}=0.65 \mathrm{~mm}$ for the forte one. Fig. 23 shows the time response of the accelerations associated with the three displacements $w_{1}, w_{2}$ and $w_{3}$. One can observe that the correspondence between the model and the experiment is particularly impressive. All the main nonlinear features are recovered by the model:

- the amplitudes of all the harmonics in both cases,

- the time delay in the response of $w_{2}$ for the weak strike,

- the amplitude modulation in both cases and its increasing frequency when the strike is stronger.

This shows that the model identified from the forced response is robust and able to reproduce with an excellent accuracy the main dynamical characteristics of an impacted note played in usual condition. Once again, this shows that a 1:2:4 internal resonance scenario is the basic ingredient for understanding the nonlinear phenomena in steelpan vibrations, a scenario that is generally complexified by the appearance of degenerate modes, as well as some higher-modes couplings with, e.g. modes at $6 f_{1}$ or $8 f_{1}$.

\section{Conclusion}

In this paper, a detailed analysis of nonlinear vibrations of a double second steelpan has been performed. The modal analysis underlines that for the two notes under study, the first modes were tuned so that their frequencies share a 1:2:4 relationship. The pattern of harmonic relationships then deflects a bit from perfect commensurable ratios, however modes are generally found around important selected harmonics such as near $6 f_{1}$ or $8 f_{1}$. An important result from our analysis, that were not particularly stressed in previous investigations on the steelpan, is the appearance of degenerate modes from the second one, so that the usual pattern should 
generally be of (at least) a 1:2:2:4:4 tuning for the first frequencies. This degeneracy is a consequence of mode localization and has already been observed in simpler systems.

The forced responses have been then studied in order to properly reveal, with a fine-tuned and reproducible experiment, the nonlinear couplings and the energy exchanges observable between modes. It has been underlined that mode couplings are numerous, leading to complex dynamical responses even for small amplitudes of vibrations, hence underlining that these couplings are necessarily activated in usual playing conditions. For small levels of excitation, simple couplings scenario invoking variations on the $1: 2: 4$ resonance are easily recognized. For higher vibration amplitudes, frequency response curves becomes complicated with a significant contribution of the cubic nonlinearity, the presence of more modes involved in the vibrations, and the appearance of quasiperiodic regimes. The main conclusion is that in steelpan's vibrations where numerous modes are tuned and thanks to the geometric nonlinearity, complex response involving numerous modes up to eight times the frequency are frequent.

Thanks to two simple models displaying $1: 2: 2$ and $1: 2: 4$ internal resonance, for which analytical solutions remains tractable, frequency response curves have been fitted. This allows to better characterize the resonance scenario, as well as to produce numerical values for the nonlinear quadratic coupling terms that can be used for time domain synthesis. This has been realized in the last section, where the identified 1:2:4 model has been used to compute time domain response of an impacted note, revealing a perfect agreement for the behaviour of the first four harmonics.

These results obviously identify the nonlinear couplings in steelpan vibration as the key to understand the rapid build-up of energy from low to high-frequencies. Two features are necessary: (i) the fact that the modes are well tuned so that $1: 2: 4: 8$ resonance are present between the eigenfrequencies, (ii) the presence of geometric nonlinearity allowing activation of energy exchanges through quadratic nonlinear terms. The simple models used in the last section can also be used within the realm of sound synthesis for producing steelpan-like sounds. This study underlines that simple models with quadratic nonlinear terms, and a sufficient number of modes (e.g. 1:2:2:4:8 resonance) should be sufficient for producing interesting sounds with a reduced order models comprising a few oscillators. In particular, such simple models should be achievable in real-time synthesis nowadays.

\section{Acknowledgement}

The authors are grateful to Bertrand David (Telecom-ParisTech) for computing the code allowing the STFT filtering procedure used in Section 5.1. The filter has been designed in the framework of the PAFI project (Plateforme d'Aide la facture Instrumentale, www. pafi.fr) which is also thanked.

\section{Appendix A. Transverse displacement expression $w_{2}$ for the 1:2:2 internal resonance model}

The amplitude $w_{2}$ and phase $\psi_{2}$ of the second harmonics of displacement at the measured point, as a function of the model variables, can be obtained by identifying Eqs. (2) and (3). Using complex numbers manipulations, one finally obtains:

$w_{2}=\sqrt{\Phi_{2}^{2} a_{2}^{2}+\Phi_{3}^{2} a_{3}^{2}+\Phi_{2} \Phi_{3} a_{2} a_{3} \cos \left(\varphi_{3}-\varphi_{2}\right)}$,

and

$\psi_{2}=\varphi_{2}+\theta$ where

$\theta=\operatorname{atan}\left(\frac{\Phi_{3} a_{3} \sin \left(\varphi_{3}-\varphi_{2}\right)}{\Phi_{2} a_{2}+\Phi_{3} a_{3} \cos \left(\varphi_{3}-\varphi_{2}\right)}\right)$

\section{References}

[1] U. Kronman, Steel Pan Tuning: A Handbook for Steel Pan Making and Tuning, Musikmuseet skrifter, iSSN 0282-8952, 1992

[2] Achong A. The steelpan as a system of non-linear mode-localized oscillators, I: theory, simulations, experiments and bifurcations. J. Sound Vib. 1996;197(4):471-87.

[3] Achong A, Sinanan-Singh KA. The steelpan as a system of non-linear modelocalized oscillators, Part II: coupled sub-systems, simulations and experiments. J. Sound Vib. 1997;203(4):547-61.

[4] Achong A. The steelpan as a system of non-linear mode-localized oscillators, part III: the inverse problem-parameter estimation. J. Sound Vib. 1998;212(4):623-35.

[5] Achong A. Non-linear analysis of compressively/thermally stressed elastic shell structures on the steelpan and the underlying theory of the tuning process. J. Sound Vib. 1999;222(4):597-620.

[6] Rossing TD, Hampton DS, Hansen UJ. Music from oil drum: the acoustics of the steel pan. Phys. Today 1996(March):24-9.

[7] Rossing TD, Hansen UJ, Hampton DS. Vibrational mode shapes in Caribbean steelpans. I. Tenor and double second. J. Acoust. Soc. Am. 2000;108(2):803-12.

[8] Rossing TD, Hansen UJ. Vibrational mode shapes in Caribbean steelpans. II. Cello and bass. Appl. Acoust. 2004;65:1233-47.

[9] Copeland B, Morrison A, Rossing TD. Sound radiation from Caribbean steelpans. J. Acoust. Soc. Am. 2005;117(1):375-83.

[10] Muddeen F, Copeland B. Sound radiation from Caribbean steelpans using nearfield acoustical holography. J. Acoust. Soc. Am. 2012;131(2):1558-65.

[11] Ryan T, O'Malley P, Glean A, Vignola J, Judge J. Conformal scanning laser Doppler vibrometer measurement of tenor steelpan response to impulse excitation. J. Acoust. Soc. Am. 2012;132(5):3494-501.

[12] É. Bavu, C. Auzou, M. Monteil, M. Melon, C. Langrenne, A. Garcia, Time-reversal imaging and field-separation-method applied to the study of the steelpan radiation, in: Proceedings of Acoustics 2012 Conference, Nantes, France, 2012.

[13] Murr LE, Ferreyra E, Maldonado J, Trillo EA, Pappu S, Kennedy C, et al. deformation phenomena and acoustic fundamentals. J. Mater. Sci. 1999;34:967-79.

[14] Ferreyra E, Maldonado J, Murr LE, Pappu S, Trillo EA, Kennedy C, et al. hardness profiles and tuning effects. J. Mater. Sci. 1999;34:981-96.

[15] Ferreyra E, Murr L. Metallography in music. Microstructures in the Caribbean steel drum. Mater. Character. 2000;45:341-51.

[16] Ferreyra E, Murr L, Russel DP, Bingert J. Elastic interactions and the metallurgical and acoustic effects of carbon in the Caribbean steel drum. Mater. Character. 2001;47:325-63.

[17] Murr L, Esquivel E, Bujanda A, Martinez K, Soto NE, Tapia A, et al. Metallurgical and acoustical comparisons for a brass pan with a Caribbean steelpan standard. J. Mater. Sci. 2004;39:4139-55.

[18] Murr L, Esquivel E, Lawrie S, Lopez M, Lair S, Soto K, et al. Musical pan: metallurgical and acoustical characterization. Mater. Character. 2006;57:232-43.

[19] Murr L, Gaytan S, Lopez M, Bujanda D, Martinez E, Whitmyre G, et al. Materials science and metallurgy of the Caribbean steel drum. Part I: fabrication, deformation phenomena and acoustic fundamentals. Mater. Character. 2008;59:321-8.

[20] Monteil M, Touzé C, Thomas O, Benacchio S. Nonlinear forced vibrations of thin structures with tuned eigenfrequencies: the cases of $1: 2: 4$ and 1:2:2 internal resonances. Nonlin. Dynam. 2014;75(1-2):175-200.

[21] M. Monteil, Vibrations Non Linéaires de Steeldrums, Caractérisation Expérimentale et Modèle Phénoménologique, Master's thesis, Université Pierre et Marie Curie, in French, 2010.

[22] Thomas O, Touzé C, Chaigne A. Asymmetric non-linear forced vibrations of free-edge circular plates. Part 2: experiments. J. Sound Vib. 2003;265:1075-101.

[23] Thomas O, Touzé C, Luminais E. Non-linear vibrations of free-edge thin spherical shells: experiments on a 1:1:2 internal resonance. Nonlin. Dynam. 2007;49(1-2):259-84.

[24] Luongo A. Mode localization in dynamics and buckling of linear imperfect continuous structures. Nonlin. Dynam. 2001;25:133-56.

[25] Nayfeh A, Mook D. Nonlinear Oscillations. John Wiley \& Sons, Inc.; 1979.

[26] Nayfeh T, Asrar W, Nayfeh A. Three-mode interactions in harmonically excited systems with quadratic nonlinearities. Nonlin. Dynam. 1992;3:385-410.

[27] Nayfeh AH. Nonlinear Interactions. John Wiley and Sons; 2000.

[28] Haddow A, Barr A, Mook D. Theoretical and experimental study of modal interaction in two-degree-freedom structure. J. Sound Vib. 1984;97(3):451-73.

[29] Shampine LF, Reichelt MW. The Matlab ODE suite. SIAM J. Scient. Comput. 1997:18(1):1-22. 